\title{
Generating family invariants for Legendrian links of unknots
}

\author{
JILL JORDAN \\ LISA TRAYNOR
}

\begin{abstract}
Theory is developed for linear-quadratic at infinity generating families for Legendrian knots in $\mathbb{R}^{3}$. It is shown that the unknot with maximal Thurston-Bennequin invariant of -1 has a unique linear-quadratic at infinity generating family, up to fiber-preserving diffeomorphism and stabilization. From this, invariant generating family polynomials are constructed for 2-component Legendrian links where each component is a maximal unknot. Techniques are developed to compute these polynomials, and computations are done for two families of Legendrian links: rational links and twist links. The polynomials allow one to show that some topologically equivalent links with the same classical invariants are not Legendrian equivalent. It is also shown that for these families of links the generating family polynomials agree with the polynomials arising from a linearization of the differential graded algebra associated to the links.
\end{abstract}

53D10; 57M25

\section{Introduction}

A basic problem in contact topology is determining when two Legendrian knots or links are equivalent. Two Legendrian links that are topologically equivalent can sometimes be distinguished via the classical Legendrian invariants of the rotation and ThurstonBennequin numbers for the components. In recent years, new invariants for Legendrian links have come from Legendrian contact homology, (see Chekanov [3], Etnyre, $\mathrm{Ng}$ and Sabloff [10], and Eliashberg, Givental and Hofer [6]). One can use the theory of holomorphic curves to associate a differential, graded algebra (DGA) to a Legendrian link. It is sometimes possible to associate an invariant polynomial to a Legendrian link by means of this DGA (see, for example, [3], $\mathrm{Ng}$ [12], and $\mathrm{Ng}$ and Traynor [13]).

New invariants for some Legendrian links have also come from the theory of generating families. The theory of generating families, also known as generating functions, is quite classic; some history can be found, for example, in Eliashberg and Gromov [7]. In the early 1990's, the technique of generating functions received renewed attention due to the work of Viterbo [20]. Viterbo found that the 0 -section of a cotangent bundle 
has a unique quadratic at infinity generating family. A careful proof of this uniqueness statement was carried out by David Théret in [15]. This uniqueness result has many interesting symplectic applications; see, for example, [20], Theŕet [16], and Traynor [17]. By some standard identifications, this uniqueness result leads to a uniqueness result for quadratic at infinity generating functions of the Legendrian 1-jet of the zero function in $\mathcal{J}^{1}(M)$. From this uniqueness result in the contact setting, it is possible to construct invariant polynomials for two component links in $\mathcal{J}^{1}\left(S^{1}\right)$ when each component is Legendrian isotopic to the 1-jet of a function $f: S^{1} \rightarrow \mathbb{R}$ (see Traynor [19]).

The particular focus of this paper is to associate a pair of invariant polynomials to a specific type of two-component Legendrian link, one in which each component is a Legendrian unknot with maximal Thurston-Bennequin number of -1 . We will call such an unknot a maximal unknot. Figure 1 shows a maximal unknot; the image of this under any contact isotopy will also be a maximal unknot.

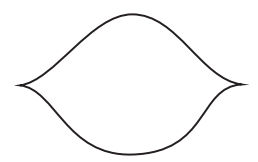

Figure 1: A maximal Legendrian unknot

We will now look at two families of Legendrian links that can be constructed from maximal unknots. The first type, called a rational link, is topologically a closure of a rational tangle, and we use a vector notation to describe it. Similar notations for rational tangles and for another type of Legendrian link are found in Ernst [8] and Traynor [19], respectively. We define the link $L=\left(2 w_{n}, k_{n}, \ldots, 2 w_{1}, k_{1}, 2 w_{0}\right), w_{i}, k_{i}>0$, recursively as follows. For $n=0$, the link has $2 w_{0}$ "horizontal" crossings, as illustrated in Figure 2 for the case $w_{0}=2$. For $n \geq 1$, the link $\left(2 w_{n}, k_{n}, \ldots, 2 w_{1}, k_{1}, 2 w_{0}\right)$ is formed from the link $\left(2 w_{n}, k_{n}, \ldots, 2 w_{1}\right)$ by adding $k_{1}$ "vertical" crossings and $2 w_{0}$ "horizontal" crossings as shown in Figure 3. For example, see Figure 4 to see how we build the link $(4,2,2,1,2)$ from the link $(4,2,2)$.

We will call a second type of link under consideration a twist link. Topologically, twist links are formed by clasping together two unknots, twisting each component a number of times, and then clasping the knots together again. We will let $L_{j, k}=\left(\Lambda_{1}, \Lambda_{0}\right)$ represent the twist link with left component $\left(\Lambda_{1}\right)$ twisted so it has $j$ crossings, and right component $\left(\Lambda_{0}\right)$ twisted to have $k$ crossings; see Figure 5. 


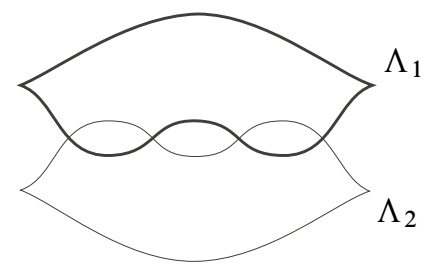

Figure 2: The Legendrian link $L=(4)$

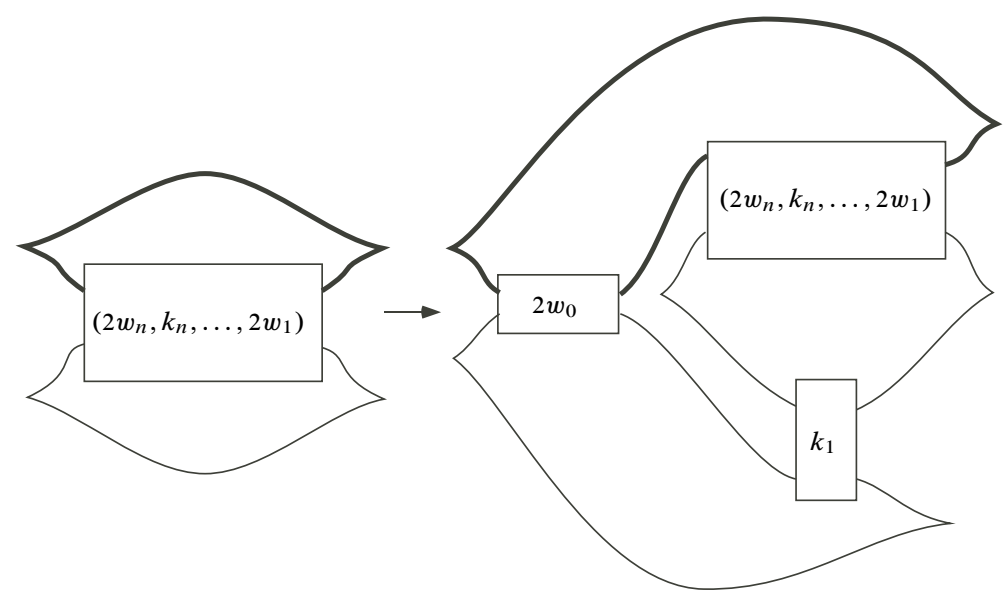

Figure 3: The link $\left(2 w_{n}, k_{n}, \ldots, 2 w_{1}, k_{1}, 2 w_{0}\right)$ is built from the link $\left(2 w_{n}, k_{n}, \ldots, 2 w_{1}\right)$.

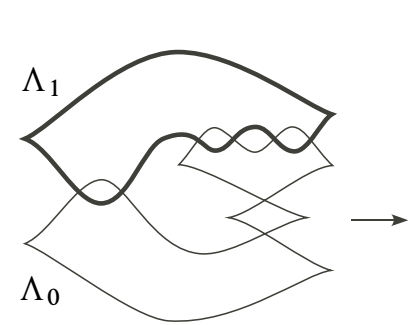

$(4,2,2)$

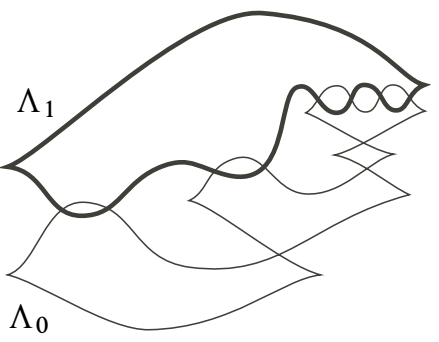

$(4,2,2,1,2)$

Figure 4: The link $(4,2,2,1,2)$ is built from the link $(4,2,2)$.

These links of maximal unknots will be studied via the technique of generating families. The type of generating families we use to study these links is different from the generating families used in Traynor [19] to study links of topologically nontrivial components in $\mathcal{J}^{1}\left(S^{1}\right)$. Previously Traynor associated a quadratic at infinity generating family to each link component $\Lambda_{i}$. That is, given $\Lambda_{i}$, there exists a function $F_{i}: S^{1} \times \mathbb{R}^{N_{i}} \rightarrow \mathbb{R}$ 


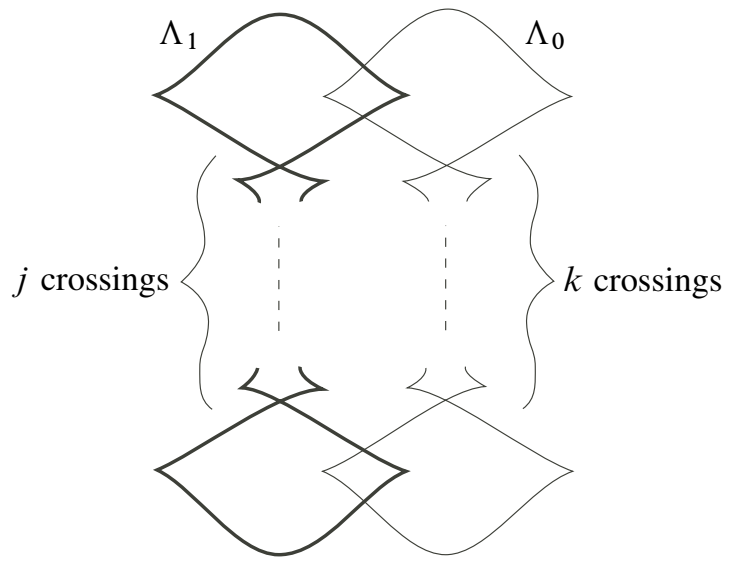

Figure 5: The twist link $L_{j, k}$

for some $N_{i} \geq 0$, such that

$$
\begin{gathered}
\Lambda_{i}=\left\{\left(x, \partial_{x} F_{i}(x, v), F_{i}(x, v)\right) \mid \partial_{v} F_{i}(x, v)=0\right\} \text { and } \\
F_{i}(x, v) \equiv Q(v) \text { outside a compact set, }
\end{gathered}
$$

where $Q$ is a nondegenerate quadratic function. However, it is not hard to see that when a component $\Lambda_{i}$ is a knot in $\mathbb{R}^{3}$, it cannot be defined by a function that is quadratic at infinity. We will see that it is sometimes possible to define a Legendrian knot by a "linear-quadratic" at infinity generating family, abbreviated as an LQ generating family. A function $F_{i}: \mathbb{R} \times\left(\mathbb{R} \times \mathbb{R}^{N_{i}}\right) \rightarrow \mathbb{R}$ is an LQ generating family for $\Lambda_{i} \subset \mathcal{J}^{1}(\mathbb{R})=\mathbb{R}^{3}$ if

$$
\begin{gathered}
\Lambda_{i}=\left\{\left(x, \partial_{x} F_{i}(x, l, v), F_{i}(x, l, v)\right) \mid \partial_{l} F_{i}(x, l, v)=0 \text { and } \partial_{v} F_{i}(x, l, v)=0\right\} \\
\text { and } F_{i}(x, l, v) \equiv J(l)+Q(v) \text { outside a compact set, }
\end{gathered}
$$

where $J$ is a nonzero linear function and $Q$ is a nondegenerate quadratic function.

In order to use LQ generating families to construct polynomial invariants, we need existence and uniqueness results. The following three theorems, proved in Section 3, are modeled after David Théret's results in the symplectic category [15].

Theorem 1.1 (Existence Theorem) Let $\Lambda$ be a maximal Legendrian unknot. Then $\Lambda$ has an $L Q$ generating family.

An LQ generating family can be explicitly constructed for some configurations of a maximal unknot, such as the one shown in Figure 1. Since any maximal unknot can be 
isotoped to look like the one in Figure 1, the existence theorem is a direct result of the following theorem, in the case where $\Delta_{n}$ is zero dimensional.

Theorem 1.2 (Serre Fibration Structure Theorem) (See Theorem 3.3) Let $\mathcal{F}$ be the set of $L Q$ generating families and let $\mathcal{L}$ be the set of Legendrian submanifolds of $\mathcal{J}^{1}(M)$. Then the map $\pi: \mathcal{F} \rightarrow \mathcal{L}$ is a smooth Serre fibration, up to equivalence. More precisely, if the smooth map $f: \Delta_{n} \rightarrow \mathcal{L}$ has a smooth lift $F: \Delta_{n} \rightarrow \mathcal{F}$ and if $\left(f_{t}: \Delta_{n} \rightarrow \mathcal{L}\right)_{t \in[0,1]}$ is a smooth homotopy of $f=f_{0}$, then there is a smooth homotopy $\left(F_{t}: \Delta_{n} \rightarrow \mathcal{F}\right)_{t \in[0,1]}$ such that $F_{0}=F$ up to equivalence (that is, up to stabilization and fiber-preserving diffeomorphism), and $\pi \circ F_{t}=f_{t}$ for every $t \in[0,1]$.

It is clear that, if a LQ generating family exists, it is not unique. However it is unique up to a certain equivalence.

Theorem 1.3 (Uniqueness Theorem) (See Theorem 3.6.) Let $\Lambda$ be a maximal Legendrian unknot. Then all $L Q$ generating families for $\Lambda$ are equivalent up to stabilization and fiber-preserving diffeomorphism.

The operations of stabilization and fiber-preserving diffeomorphism are explained in Definition 2.1.

In Section 4, in analogy with [19], we apply the existence and uniqueness results from Section 3 to associate polynomials, $\Gamma_{\Delta}^{ \pm}(\lambda)$, to a two-component Legendrian link where each component is a maximal unknot. Normalized versions of these polynomials give Laurent polynomials $\Gamma^{ \pm}(\lambda)$ that are shown to be invariants of a Legendrian link. In fact, $\Gamma^{+}(\lambda)$ is determined by $\Gamma^{-}(\lambda): \Gamma^{+}(\lambda)=\lambda \cdot \Gamma^{-}(\lambda)$. The following theorems give the calculations of $\Gamma^{-}$for rational links and twist links.

Theorem 1.4 (See Theorem 5.4) Let $L$ be the Legendrian link

$$
\left(2 w_{n}, k_{n}, \ldots, 2 w_{1}, k_{1}, 2 w_{0}\right)
$$

as described in Figure 3. Then

$$
\Gamma^{-}(\lambda)[L]=w_{0} \lambda^{0}+w_{1} \lambda^{-k_{1}}+w_{2} \lambda^{-\left(k_{1}+k_{2}\right)}+\cdots+w_{n} \lambda^{-\left(k_{1}+k_{2}+\cdots+k_{n}\right)} .
$$

Theorem 1.5 (See Theorem 5.9) Let $L_{j, k}$ be a Legendrian twist link as described in Figure 5. Then

$$
\Gamma^{-}(\lambda)\left[L_{j, k}\right]=\lambda^{0}+\lambda^{-|j-k|} .
$$


The topological link type of a twist link is dependent only on the value of $j+k$. So as a direct result of Theorem 1.5, we are able to use generating family polynomials to distinguish several Legendrian twist links with the same topological link type; see Corollary 5.11.

Generating family polynomials can also be used to show that a link is ordered. $L=$ $\left(\Lambda_{1}, \Lambda_{0}\right)$ is ordered if it is not equivalent to $\bar{L}=\left(\Lambda_{0}, \Lambda_{1}\right)$. In fact, a necessary condition for a link to be unordered is that the $\Gamma^{-}$polynomial must be the same, up to a shift, if $\lambda$ is replaced by $\lambda^{-1}$.

Theorem 1.6 (See Theorem 4.11) Let $L=\left(\Lambda_{1}, \Lambda_{0}\right)$ be a Legendrian link where $\Lambda_{1}$ and $\Lambda_{0}$ are maximal unknots. If there does not exist an $l \in \mathbb{Z}$ such that $\Gamma^{-}(\lambda)[L]=$ $\lambda^{l} \cdot \Gamma^{-}\left(\lambda^{-1}\right)[L]$, then the link $L$ is ordered.

The rational links are always topologically unordered, but by the above polynomial calculations we show:

Theorem 1.7 (See Corollary 5.6) Let $L=\left(\Lambda_{1}, \Lambda_{0}\right)$ be the rational Legendrian link $\left(2 w_{n}, k_{n}, \ldots, 2 w_{1}, k_{1}, 2 w_{0}\right)$. If the vector $\left(2 w_{n}, k_{n}, \ldots, 2 w_{1}, k_{1}, 2 w_{0}\right)$ is not palindromic, then the link $L$ is ordered.

Another use of generating family polynomials is in distinguishing rational links that differ by a Legendrian "flyping" operation. Flypes are discussed in Section 5. Both horizontal and vertical flyping procedures, when applied to a Legendrian link, result in a link of the same topological link type. It is shown by Traynor [19] that vertical flypes when applied to a Legendrian link $L=\left(2 w_{n}, k_{n}, \ldots, 2 w_{1}, k_{1}, 2 w_{0}\right)$ preserve the Legendrian link type. However polynomial calculations for horizontal flypes show that nonequivalent Legendrian links may be produced.

Theorem 1.8 (See Theorem 5.7) Let $L=\left(2 w_{n}, k_{n}, 2 w_{n-1}^{p_{n-1}}, \ldots, k_{1}, 2 w_{0}^{p_{0}}\right)$ be the Legendrian link obtained by doing $p_{i}$ horizontal flypes to the $w_{i}$ horizontal entry of the rational link $\left(2 w_{n}, k_{n}, \ldots, k_{1}, 2 w_{0}\right)$. For $j=0,1, \ldots, n-1$, let $\sigma(j)=1+\sum_{i=0}^{j} p_{i}$ mod 2. Then

$$
\Gamma^{-}(\lambda)[L]=\lambda^{m} \cdot\left[w_{0} \lambda^{0}+\sum_{i=1}^{n} w_{i} \lambda^{\left[(-1)^{\sigma(0)} k_{1}+\cdots+(-1)^{\sigma(i-1)} k_{i}\right]}\right],
$$

where $m$ is chosen so that $\Gamma^{-}$has degree zero.

For an example of a rational link that is not equivalent to a flyped version of the link, see Figure 6. 


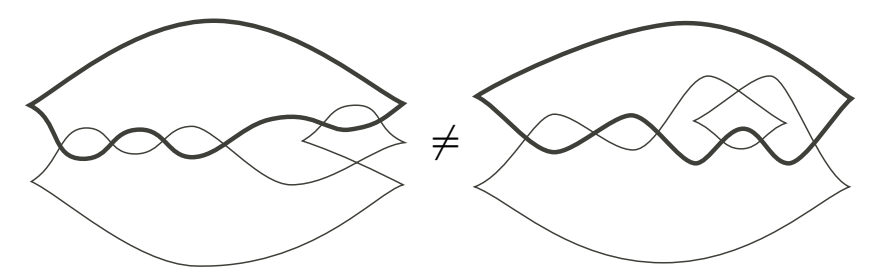

Figure 6: The link $(2,1,4)$ is not equivalent to its flyped version, $\left(2,1,4^{1}\right)$

Finally, we compare generating family polynomials to decomposition number polynomials and homology polynomials obtained from the DGA. The decomposition number polynomials are quite different from the generating function polynomials. However, for both rational links and twist links, the (negative) generating family polynomials are the same as the (negative) DGA homology polynomials; see Theorem 6.4.

Acknowledgements We are thankful to the members of the Philadelphia Area Contact Topology (PACT) Seminar for their useful comments during a mini-series presentation of these results. We are also grateful for the many careful and detailed suggestions of the reviewer.

\section{Background information}

If $M$ is an $n$-manifold, then the 1 -jet space of $M, \mathcal{J}^{1}(M)=T^{*}(M) \times \mathbb{R}$, is a $(2 n+1)$-manifold with a contact structure $\xi_{\text {std }}$ on $\mathcal{J}^{1}(M)=\{(x, y, z)\}$ given by the kernel of the 1 -form $\alpha=d z-y d x$. There are no integral $j$-dimensional submanifolds of the $(2 n+1)$-dimensional $\mathcal{J}^{1}(M)$ when $j>n$. However, there are numerous integral $n$-dimensional submanifolds. Such submanifolds are called Legendrian. For example, for any $f: M \rightarrow \mathbb{R}, j^{1}(f):=\left\{\left(x, \frac{\partial f}{\partial x}, f(x)\right) \mid x \in M\right\}$ is a Legendrian submanifold of $\mathcal{J}^{1}(M)$. We will pay special attention to the 3 -dimensional contact manifold $\mathcal{J}^{1}(\mathbb{R})=\mathbb{R}^{3}$.

A Legendrian knot is a closed and connected 1-dimensional Legendrian submanifold in a 3-dimensional contact manifold. A Legendrian link is the union of one or more non-intersecting Legendrian knots. The front of a Legendrian curve is its image under the front projection given by $\pi_{x z}(x, y, z)=(x, z)$. Given a Legendrian curve $L$ in $\mathcal{J}^{1}(\mathbb{R})$, let $\pi_{x z}(L)=C . C$ is an immersed curve with nonvertical tangents and semi-cubic cusps (see Figure 7), which generically has only double points. If $L$ is free of self-intersections, any crossing of $C$ must be a transverse intersection. Conversely, any such curve in $\mathbb{R}^{2}$ determines a Legendrian curve in $\mathbb{R}^{3}$. We may use the fact that 
$y=\frac{d z}{d x}$ for any $(x, y, z) \in L$, a condition imposed by the contact structure, to recover the third coordinate of $L$.

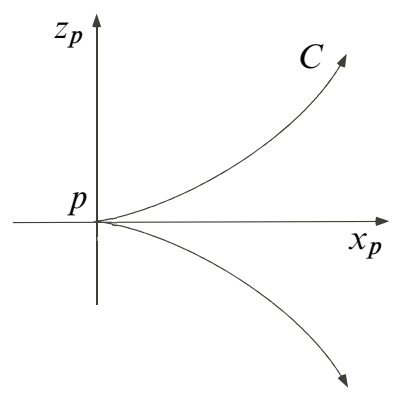

Figure 7: A semi-cubic cusp is a point $p \in C$ for which there exist coordinates $x_{p}, z_{p}$ such that $p=(0,0)$ and $C=\left\{\left(x_{p}, z_{p}\right) \mid z_{p}^{2}= \pm x_{p}^{3}\right\}$ in a neighborhood of $p$.

The main question in the study of Legendrian links is whether or not two links are equivalent. We say two links $L=\left(\Lambda_{n}, \ldots, \Lambda_{0}\right), \widetilde{L}=\left(\widetilde{\Lambda}_{n}, \ldots, \widetilde{\Lambda}_{0}\right)$ are equivalent if there exists a smooth, one-parameter family $L_{t}$ of Legendrian links such that $L_{0}=L$ and $L_{1}=\widetilde{L}$, or, equivalently, there exists a contact isotopy of the ambient space taking $\Lambda_{i}$ to $\widetilde{\Lambda}_{i}$ for $i=0, \ldots, n$. Two topologically equivalent Legendrian links will be distinct if, for some $i$, components $\Lambda_{i}$ and $\widetilde{\Lambda}_{i}$ do not have the same classical Legendrian integer invariants given by the rotation and Thurston-Bennequin numbers. For more background on Legendrian knots and links, see, for example, Etnyre [9]. In the following, we will extend the work in [19] and construct new invariants for Legendrian links using the technique of generating families.

Generating families provide a way to encode a Legendrian submanifold of $\mathcal{J}^{1}(M)$ by a real-valued function on $M \times \mathbb{R}^{k}$. Suppose we have a smooth function $F: M \times \mathbb{R}^{k} \rightarrow \mathbb{R}$, $(x, v) \mapsto F(x, v)$, such that 0 is a regular value of the map $\left(\frac{\partial F}{\partial v_{1}}, \ldots, \frac{\partial F}{\partial v_{n}}\right): M \times \mathbb{R}^{k} \rightarrow$ $\mathbb{R}^{k}$. We define $\Sigma_{F}$, the critical locus of $F$, as

$$
\Sigma_{F}:=\left\{(x, v) \in M \times \mathbb{R}^{k} \mid \frac{\partial F}{\partial v_{i}}(x, v)=0 \text { for } i=1,2, \ldots, k\right\} .
$$

By the preimage theorem, $\Sigma_{F}$ is a one-dimensional submanifold of $M \times \mathbb{R}^{k}$. Define an immersion $i_{F}: \Sigma_{F} \rightarrow \mathcal{J}^{1}(M)$ by

$$
i_{F}\left(x_{0}, v_{0}\right)=\left(x_{0}, \partial_{x} F\left(x_{0}, v_{0}\right), F\left(x_{0}, v_{0}\right)\right) .
$$

When $i_{F}$ is an embedding, $L:=i_{F}\left(\Sigma_{F}\right)$ is a Legendrian submanifold of $\mathcal{J}^{1}(M)$. We say that $F$ generates $L$, or $F$ is the generating family for $L$. In the following, we 
will start with a Legendrian submanifold $L \subset \mathcal{J}^{1}(M)$ and seek a generating family $F: M \times \mathbb{R}^{k} \rightarrow \mathbb{R}$ for $L$.

Clearly the choice of a generating family for a given Legendrian $L \subset \mathcal{J}^{1}(M)$, if one exists, is not unique. If $F: M \times \mathbb{R}^{k} \rightarrow \mathbb{R}$ generates $L$ then so does, for example, $F^{\prime}: M \times \mathbb{R}^{k+1} \rightarrow \mathbb{R}$, where $F^{\prime}\left(x, v_{1}, \ldots, v_{k}, v_{k+1}\right)=F\left(x, v_{1}, \ldots, v_{k}\right)+v_{k+1}^{2}$. Therefore we wish to work with equivalence classes of families rather than with the families themselves.

Definition 2.1 Two generating families $F_{i}: M \times \mathbb{R}^{k_{i}} \rightarrow \mathbb{R}, i=1,2$ are equivalent if and only if they can be made equal after a succession of fiber-preserving diffeomorphisms and stabilizations; these operations on the generating family are defined as follows:

(1) Given a generating family $F: M \times \mathbb{R}^{k} \rightarrow \mathbb{R}$, suppose $\Phi: M \times \mathbb{R}^{k} \rightarrow M \times \mathbb{R}^{k}$ is a fiber-preserving diffeomorphism, i.e., $\Phi(x, v)=\left(x, \phi_{x}(v)\right)$ for diffeomorphisms $\phi_{x}$. Then $F^{\prime}=F \circ \Phi$ is said to be obtained from $F$ by a fiber-preserving diffeomorphism.

(2) Given a generating family $F: M \times \mathbb{R}^{k} \rightarrow \mathbb{R}$, let $Q: \mathbb{R}^{j} \rightarrow \mathbb{R}$ be a quadratic function. Define $F^{\prime}: M \times \mathbb{R}^{k} \times \mathbb{R}^{j} \rightarrow \mathbb{R}$ by $F^{\prime}\left(x, v_{1}, v_{2}\right)=(F \oplus Q)\left(x, v_{1}, v_{2}\right)=$ $F\left(x, v_{1}\right)+Q\left(v_{2}\right)$. Then $F^{\prime}$ is said to obtained from $F$ by a stabilization.

If two families are equivalent, we can get from one to another by performing one stabilization followed by one diffeomorphism (see Théret [15]).

There is a parallel theory of generating families in the symplectic category. In a symplectic manifold, Lagrangian submanifolds are objects of central importance, and the theory of generating families gives one a way to encode some Lagrangians in $T^{*}(M)$ by a function $F: M \times \mathbb{R}^{N} \rightarrow \mathbb{R}$. In this version, the same procedure is used to construct $\sigma_{F}$, but now the associated immersion $i_{F}$ does not include the value of $F$.

In both the contact and symplectic settings, these generating families are defined on noncompact domains. Analytically it is convenient to consider functions that are "well-behaved" outside of a compact set. A common convention has been to consider generating families that are "quadratic at infinity." This means that outside of a compact set, $F(x, v)=Q(v)$, where $Q$ is a nondegenerate quadratic function. See, for example, Viterbo [20] and Théret [15].

Quadratic at infinity generating families can generate only particular Legendrian or Lagrangian submanifolds. It is not hard to see, for example, that the maximal Legendrian unknot pictured in Figure 1 does not have a quadratic at infinity generating family. However, this Legendrian knot will have a "linear-quadratic" at infinity generating family, abbreviated as an LQ generating family. 
Definition 2.2 A generating family $F: M \times \mathbb{R} \times \mathbb{R}^{k} \rightarrow \mathbb{R}, k \geq 0$ is linear-quadratic at infinity if we have $F(x, l, v)=J_{x}(l)+Q_{x, l}(v)$ outside a compact set, where $J_{x}$ is a nonzero linear function of $l$ for each $x$ and $Q_{x, l}$ is a nondegenerate quadratic function of $v$ for each pair $(x, l) \in M \times \mathbb{R} . F$ is special linear-quadratic at infinity if $F(x, l, v)=J(l)+Q(v)$ outside a compact set for some nonzero linear function $J$ and some nondegenerate quadratic function $Q$.

Note that the added requirement for a special LQ generating family is that the linear and quadratic parts be independent of the base point $x \in M$. In fact, any LQ generating family is equivalent to a special LQ generating family. This can be proved following the argument of the proof of [15, Proposition 2.12].

Figure 8 sketches the graphs of two LQ generating families for the maximal Legendrian unknot. Note that the generating families differ by a stabilization, and hence they are equivalent.

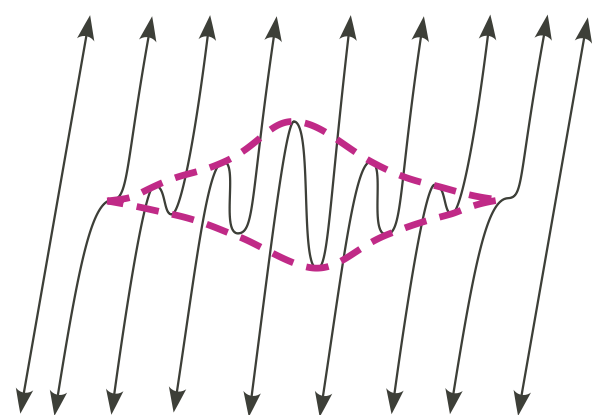

(a)

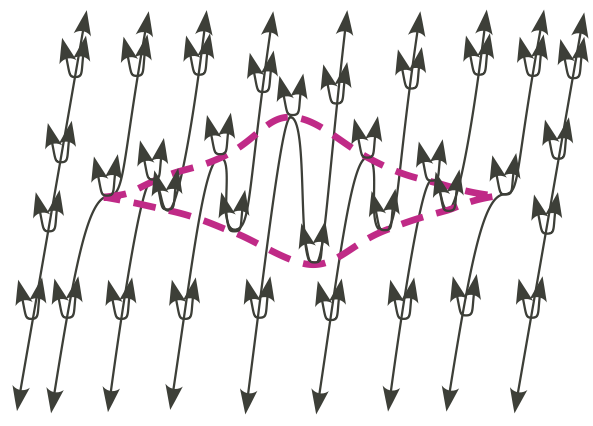

(b)

Figure 8: The LQ generating families shown in (a) and (b) generate the same maximal unknot, which is sketched in with a broken line. The generating family in (b) is a stabilization of the one in (a).

\section{Existence and uniqueness of LQ generating families}

In Sections 3.1 and 3.2, we prove that if we have a Legendrian $L \subset \mathcal{J}^{1}\left(\mathbb{R}^{m}\right)$ with a unique LQ generating family then $\varphi_{1}(L)$ also has a unique LQ generating family for any contact isotopy $\varphi_{t}$ of $\mathcal{J}^{1}\left(\mathbb{R}^{m}\right)$. SubSection 3.3 contains the proof of uniqueness of an LQ generating family for the maximal unknot in $\mathcal{J}^{1}(\mathbb{R})=\mathbb{R}^{3}$ pictured in Figure 1. Théret's work in [15] forms a basis for the theorems and proofs in this section. 


\subsection{Persistence of LQ generating families under isotopies}

Let us first introduce some notation for use in this subsection and the next. Let $M$ be $\mathbb{R}^{m}$ or $\Delta_{n} \times \mathbb{R}^{m-n} \subset \mathbb{R}^{m}$ where $\Delta_{n}$ is the standard $n$-simplex in $\mathbb{R}^{n}$. Results in this section will also apply to the case where $M$ is a closed manifold but this setting will not be needed in this paper. Let $\mathcal{L}$ be the set of Legendrians in $\mathcal{J}^{1}(M)$. For each integer $k \geq 0$, let $\mathcal{F}_{k}$ be the set of all LQ generating families defined on $M \times \mathbb{R} \times \mathbb{R}^{k}$. If $k$ is not specified we will simply use $\mathcal{F}$. Let $\pi: \mathcal{F} \rightarrow \mathcal{L}$ be the map taking a generating family $f$ to the Legendrian in $\mathcal{L}$ generated by $f$.

The main result of this subsection is that the map $\pi: \mathcal{F} \rightarrow \mathcal{L}$ is a smooth Serre fibration (up to equivalence). In particular, this implies that if $\gamma:[0,1] \rightarrow \mathcal{L}$ is a path and $\gamma(0)$ has a generating family (i.e. there exists $\left.F \in \pi^{-1}(\gamma(0))\right)$, then there is a lift of the path to $\tilde{\gamma}:[0,1] \rightarrow \mathcal{F}$ such that $\tilde{\gamma}(0)=F$ up to equivalence and $\pi(\tilde{\gamma}(t))=\gamma(t)$ for all $t \in[0,1]$. More generally, if $f: \Delta_{n} \rightarrow \mathcal{L}$ has a smooth lift $F: \Delta_{n} \rightarrow \mathcal{F}$, then for any smooth homotopy $f_{t}: \Delta_{n} \rightarrow \mathcal{L}$ of $f$ there exists a smooth homotopy $F_{t}: \Delta_{n} \rightarrow \mathcal{F}$ of (perhaps an equivalent version of) $F$ satisfying $\pi \circ F_{t}=f_{t}$. We will prove this Serre fibration property in two stages. We will first show that the above path lifting property holds (see Theorem 3.1). This follows from a Legendrian version of "Chekanov's formula". We will then prove a lemma (Lemma 3.2) that allows us to view a homotopy of Legendrians as a single Legendrian in a larger space. Theorem 3.1 will then be applied again.

Theorem 3.1 Let $M$ be $\mathbb{R}^{m}$ or $\Delta_{n} \times \mathbb{R}^{m-n} \subset \mathbb{R}^{m}$, and let $\Lambda$ be a Legendrian submanifold of $\mathcal{J}^{1}(M)$. Let $\left(\kappa_{t}\right)_{t \in[0,1]}$ be a compactly supported contact isotopy of $\mathcal{J}^{1}(M), \kappa_{0}=\mathrm{id}$. Assume that $\Lambda$ has an LQ generating family $F: M \times \mathbb{R} \times \mathbb{R}^{k} \rightarrow \mathbb{R}$. Then there exists an integer $j \geq 0$ and a path $\left(F_{t}\right)_{t \in[0,1]}$ of $L Q$ generating families defined on $M \times \mathbb{R} \times \mathbb{R}^{k} \times \mathbb{R}^{j}$ such that

(1) $F_{0}(x, l, v, w)=F(x, l, v)+Q(w)$, where $Q$ is a nondegenerate quadratic function on $\mathbb{R}^{j}$;

(2) $F_{t}=F_{0}$ outside a compact set;

(3) $F_{t}$ generates $\kappa_{t}(\Lambda)$ for $t \in[0,1]$.

Proof [18, Theorem A.1] is an analogous theorem with quadratic (rather than linearquadratic) at infinity conditions. A careful check of the equation shows that the proof of Theorem A.1 generalizes to the LQ situation. The following is a brief summary of the proof.

The first step is to realize that even when $M=\Delta_{n} \times \mathbb{R}^{m-n}$ we can work in the setting of $\mathcal{J}^{1}\left(\mathbb{R}^{m}\right)$ rather than in $\mathcal{J}^{1}(M) . \Delta_{n} \times \mathbb{R}^{m-n}$ naturally lies in $\mathbb{R}^{m}$. By an 
extension of $F$ to $\mathbb{R}^{m} \times \mathbb{R} \times \mathbb{R}^{k}$, we have that $\Lambda$ embeds into a Legendrian submanifold $\Lambda^{m} \subset \mathcal{J}^{1}\left(\mathbb{R}^{m}\right)$. As shown in [18, Proposition A.2], $\kappa_{t}$ extends to a compactly supported contact isotopy $\kappa_{t}^{m}$ of $\mathcal{J}^{1}\left(\mathbb{R}^{m}\right)$, and to show $\kappa_{t}(\Lambda)$ has an LQ generating family it suffices to prove that $\kappa_{t}^{m}\left(\Lambda^{m}\right) \subset \mathcal{J}^{1}\left(\mathbb{R}^{m}\right)$ has an LQ generating family.

Next we translate the problem into a symplectic situation by looking at a certain $\mathbb{R}^{+}$-equivariant Lagrangian $\mathcal{L}_{\Lambda}$ in $T^{*}\left(\mathbb{R}^{m} \times \mathbb{R}^{+}\right)$that corresponds to a Legendrian $\Lambda \subset \mathcal{J}^{1}\left(\mathbb{R}^{m}\right)$ (see [18, Equation A.3]). If $\Lambda \subset \mathcal{J}^{1}\left(\mathbb{R}^{m}\right)$ has an LQ generating family, then so does $\mathcal{L}_{\Lambda} \subset T^{*}\left(\mathbb{R}^{m} \times \mathbb{R}^{+}\right)$.

Next we associate a symplectic diffeomorphism $\Psi_{\kappa}$ of $T^{*}\left(\mathbb{R}^{m} \times \mathbb{R}^{+}\right)$to a contact diffeomorphism $\kappa$ isotopic to the identity. This has a corresponding Lagrangian submanifold $\widetilde{\Gamma} \Psi_{\kappa}$ of $T^{*}\left(\mathbb{R}^{2 m+1} \times \mathbb{R}^{+}\right)$. When the diffeomorphism $\Psi_{\kappa}$ is sufficiently close to the identity, these Lagrangians have simple generating families. Then by "Chekanov's formula," generating families for $\mathcal{L}_{\Lambda}$ and $\widetilde{\Gamma} \Psi_{\kappa}$ can be "composed" to obtain a generating family for $\Psi_{\kappa}\left(\mathcal{L}_{\Lambda}\right)=\mathcal{L}_{\kappa(\Lambda)}$ (see [18, Proposition A.5] or [17, Section 4]). A careful analysis of these equations shows that if the generating family for $\mathcal{L}_{\Lambda}$ is LQ, then the family for $\Psi_{\kappa}\left(\mathcal{L}_{\Lambda}\right)$ will also be LQ. By breaking the isotopy $\kappa_{t}$ into a composition of small diffeomorphisms, this procedure produces an LQ generating family for $\mathcal{L}_{\kappa_{t}(\Lambda)}$ for all $t$. See, for example, [17, Section 4].

Lastly, [18, Proposition A.6] shows that if $G$ is an LQ generating family for $\mathcal{L}_{\Lambda} \subset$ $T^{*}\left(\mathbb{R}^{m} \times \mathbb{R}^{+}\right)$, then a "slice" of $G$ will be an LQ generating family for $\Lambda$. Hence since $\mathcal{L}_{\kappa_{t}(\Lambda)}$ has an LQ generating family for all $t \in[0,1], \kappa_{t}(\Lambda)$ has an LQ generating family, say $F_{t}$, for all $t \in[0,1]$. We can see through checking the proofs in [18] that the remaining conditions on $F_{t}$ are satisfied.

The next lemma shows, in particular, that from a family of Legendrian submanifolds of $\mathcal{J}^{1}(\mathbb{R})$ parameterized by points in $\Delta_{n}$ one can construct a single, $(n+1)$-dimensional Legendrian submanifold of $\mathcal{J}^{1}\left(\Delta_{n} \times \mathbb{R}\right)$. The lemma is the Legendrian version of [15, Lemma 4.3]. Note that when we say $S: \Delta_{n} \times M \times \mathbb{R} \times \mathbb{R}^{k} \rightarrow \mathbb{R}$ is linear-quadratic at infinity, we mean it is linear in the $\mathbb{R}$ variable and quadratic in the $\mathbb{R}^{k}$ variable.

Lemma 3.2 Note first that $\mathcal{J}^{1}\left(\Delta_{n} \times M\right) \cong T^{*}\left(\Delta_{n}\right) \times \mathcal{J}^{1}(M)$.

Let $\rho: \mathcal{J}^{1}\left(\Delta_{n} \times M\right) \rightarrow \mathcal{J}^{1}(M)$ be the associated projection.

(a) Let $f: \Delta_{n} \times B \rightarrow \mathcal{J}^{1}(M)$ be a differentiable map such that each $f_{a}$, defined by $f_{a}(s)=f(a, s)$ is a Legendrian embedding of $B$ in $\mathcal{J}^{1}(M)$. Then there is a map $v: \Delta_{n} \times B \rightarrow\left(\mathbb{R}^{n}\right)^{*}$ such that

$$
L:=\left\{\left(a, v(a, s), f_{a}(s)\right) \mid a \in \Delta_{n}, s \in B\right\} \subset T^{*}\left(\Delta_{n}\right) \times \mathcal{J}^{1}(M)
$$


is a Legendrian submanifold in $\mathcal{J}^{1}\left(\Delta_{n} \times M\right)$. Furthermore, if $S_{a}: M \times \mathbb{R} \times$ $\mathbb{R}^{k} \rightarrow \mathbb{R}$ is a smooth family of $L Q$ generating families of $L_{a}:=f(\{a\} \times B)$, then the total family $S: \Delta_{n} \times M \times \mathbb{R} \times \mathbb{R}^{k} \rightarrow \mathbb{R}$ is an $L Q$ generating family for the Legendrian $L$ given above.

(b) Conversely, if $F: \Delta_{n} \times B \rightarrow \mathcal{J}^{1}\left(\Delta_{n} \times M\right)$ is a Legendrian embedding, transversal to the spaces $W_{a}:=\{a\} \times\left(\mathbb{R}^{n}\right)^{*} \times \mathcal{J}^{1}(M)$ and having an LQ generating family $S: \Delta_{n} \times M \times \mathbb{R} \times \mathbb{R}^{k} \rightarrow \mathbb{R}$, then $L_{a}:=(\rho \circ F)(\{a\} \times M)$ is a Legendrian in $\mathcal{J}^{1}(M)$ with LQ generating family $S_{a}=S\left(\{a\} \times M \times \mathbb{R} \times \mathbb{R}^{k}\right)$.

Proof First, $\mathcal{J}^{1}\left(\Delta_{n} \times M\right) \cong T^{*}\left(\Delta_{n}\right) \times \mathcal{J}^{1}(M)$ by a reordering of coordinates as follows: $(a, x, w, y, z) \sim(a, w) \times(x, y, z)$ for $(a, w) \in T^{*}\left(\Delta_{n}\right)$ and $(x, y, z) \in$ $\mathcal{J}^{1}(M)$, since the contact form on $T^{*}\left(\Delta_{n}\right) \times \mathcal{J}^{1}(M)$ and $\mathcal{J}^{1}\left(\Delta_{n} \times M\right)$ is given by $\alpha=d z-y d x-w d a$.

To verify (a), let $f: \Delta_{n} \times B \rightarrow \mathcal{J}^{1}(M),(a, x) \mapsto f_{a}(x)$ be a differentiable map so that each $f_{a}$ is a Legendrian embedding of $B$ in $\mathcal{J}^{1}(M)$. Write $f_{a}(s)=$ $\left(f_{a}^{x}(s), f_{a}^{y}(s), f_{a}^{z}(s)\right)$. Then if we let $v(a, s)=\frac{\partial f_{a}^{z}}{\partial a}-f_{a}^{y}(s) \cdot \frac{\partial f_{a}^{x}}{\partial a}$,

$$
L=\left\{\left(a, f_{a}^{x}(s), v(a, s), f_{a}^{y}(s), f_{a}^{z}(s)\right)\right\} \subset \mathcal{J}^{1}\left(\Delta_{n} \times M\right)
$$

is a Legendrian submanifold. It is straightforward to check that if $S_{a}: M \times \mathbb{R} \times \mathbb{R}^{k} \rightarrow \mathbb{R}$ is a smooth family of LQ generating functions of $L_{a}:=f_{a}(M), a \in \Delta_{n}$ then the total function $S: \Delta_{n} \times M \times \mathbb{R} \times \mathbb{R}^{k} \rightarrow \mathbb{R}$ is an LQ generating family for $L$ as given above.

To verify (b), let $F: \Delta_{n} \times B \rightarrow \mathcal{J}^{1}\left(\Delta_{n} \times M\right)$ be a Legendrian embedding transversal to the spaces $W_{a}:=\{a\} \times\left(\mathbb{R}^{n}\right)^{*} \times \mathcal{J}^{1}(M)$ and having an LQ generating function $S: \Delta_{n} \times M \times \mathbb{R} \times \mathbb{R}^{k} \rightarrow \mathbb{R}$. For $a \in \Delta_{n}$, let $L_{a}:=(\rho \circ F)(\{a\} \times M)$ and let $S_{a}:=$ $S\left(\{a\} \times M \times \mathbb{R} \times \mathbb{R}^{k}\right)$. Let $L=F\left(\Delta_{n} \times M\right)$. Since $L$ is transversal to each $W_{a}$, $L \cap W_{a}$ is a submanifold of $\mathcal{J}^{1}\left(\Delta_{n} \times M\right)$ that projects to $L_{a} \subset \mathcal{J}^{1}(M)$. It is straight forward to show that $S_{a}$ generates $L_{a}$.

We are now ready to state and prove the main result of this subsection. The theorem and its proof are nearly identical to Théret's Theorem 4.2 and its proof in [15].

Theorem 3.3 The map $\pi: \mathcal{F} \rightarrow \mathcal{L}$ is a smooth Serre fibration, up to equivalence. More precisely, if the smooth map $f: \Delta_{n} \rightarrow \mathcal{L}$ has a smooth lift $F: \Delta_{n} \rightarrow \mathcal{F}$ and if $\left(f_{t}: \Delta_{n} \rightarrow \mathcal{L}\right)_{t \in[0,1]}$ is a smooth homotopy with $f=f_{0}$, then there is a smooth homotopy $\left(F_{t}: \Delta_{n} \rightarrow \mathcal{F}\right)_{t \in[0,1]}$ such that $F_{0}=F$ up to equivalence, and $\pi \circ F_{t}=f_{t}$ for every $t \in[0,1]$. 
Proof Applying (a) from Lemma 3.2, for each $t \in[0,1]$, the family $f_{t}\left(\Delta_{n}\right)$ parameterized by points in $\Delta_{n}$ can be used to construct a Legendrian $L_{t}$ in $\mathcal{J}^{1}\left(\Delta_{n} \times M\right)$. Thus we get a path $\bar{f}:[0,1] \rightarrow \mathcal{L}\left(\mathcal{J}^{1}\left(\Delta_{n} \times M\right)\right)$ whose initial point $\bar{f}_{0}$ admits a generating family $\bar{F}_{0}$. By part (b) of Lemma 3.2, it is sufficient to prove that we can lift the path $\bar{f}$ from the initial point $\bar{F}_{0}$. By Theorem 3.1, we can lift the path $\bar{f}$, as desired.

\subsection{Uniqueness of LQ generating families}

We say a Legendrian $L$ has the uniqueness property if any two of its LQ generating families are equivalent. Here we prove that if $L$ has the uniqueness property and $L_{1}$ is obtained from $L$ through a Legendrian isotopy, then $L_{1}$ has the uniqueness property as well. We first prove a lemma which allows us to get a path in $\pi^{-1}\left(L_{1}\right)$ between any two LQ generating families of $L_{1}$. This lemma corresponds to [15, Lemma 5.2].

Lemma 3.4 Suppose that $L_{0} \in \mathcal{L}$ is a Legendrian with the uniqueness property and that $L_{1}$ is obtained from $L$ through a Legendrian isotopy. Let $f$ and $f^{\prime}$ be two LQ generating families for $L_{1}$. Then up to equivalence, $f$ and $f^{\prime}$ can be connected by a path in $\pi^{-1}\left(L_{1}\right)$.

Since the proof is identical to that in [15], only the following sketch is given. Let

$f_{0}$ and $f_{0}^{\prime}$ be two LQ generating families for $L_{1}$. From the path of Legendrians between $L_{0}$ and $L_{1}$, we construct a contractible loop of Legendrians based at $L_{1}$. Using Theorem 3.1 (the path lifting property) and the fact that $L_{0}$ has the uniqueness property, we know this loop is covered by a path of generating families with endpoints at (equivalent versions of) $f_{0}$ and $f_{0}^{\prime}$. Since the loop is contractible, by Theorem 3.3 (with $n=1$ ) we get our desired result.

We now get the following uniqueness theorem, which corresponds to [15, Theorem 5.1].

Theorem 3.5 Let $L_{0}$ be a Legendrian with the uniqueness property. Suppose that $L_{1}=\varphi_{1}\left(L_{0}\right)$ where $\left(\varphi_{t}\right)_{t \in[0,1]}$ is a Legendrian isotopy of $\mathcal{J}^{1}(M)$. Then any two $L Q$ generating families of $L_{1}$ are equivalent.

Again, since the proof is nearly identical to the proof of [15, Theorem 5.1], we will only sketch the argument. If $f$ and $f^{\prime}$ are two LQ generating families for $L_{1}$, then we know by Lemma 3.4, up to equivalence, $f$ and $f^{\prime}$ can be connected by a path in $\pi^{-1}\left(L_{1}\right)$. Therefore it suffices to show that if $\left(f_{t}\right)_{t \in[0,1]}$ is a smooth path of LQ generating families that generate a fixed Legendrian, then there exists a fiber-preserving isotopy $\Phi_{t}$ such that $f_{t} \circ \Phi_{t}=f_{0}$ for all $t \in[0,1]$. By differentiating this equation 
with respect to $t$, we get an equation for the vector field $X_{t}$ that generates this isotopy. It is easy to find a solution for this $X_{t}$ outside the fiber critical set $\Sigma_{t}=\Sigma$ of $f_{t}$. We then apply Hadamard's Lemma to find a solution for $X_{t}$ near $\Sigma$. These two solutions are then pasted together by the choice of an appropriate bump function.

\subsection{Uniqueness of the LQ generating family for a basic unknot}

So far we have proved that existence and uniqueness of LQ generating families persist under Legendrian isotopies. We will now prove that the maximal unknot in $\mathcal{J}^{1}(\mathbb{R})$ shown in Figure 1 has the uniqueness property. The proof of this theorem is philosophically the same as [15, Theorem 6.1] where it is proved that the Lagrangian 0-section of $T^{*} M$, for $M$ an arbitrary closed manifold, has a unique quadratic at infinity generating family. Some differences between the proofs are pointed out in Remark 3.8 after the proof.

Theorem 3.6 Let $L \subset \mathcal{J}^{1}(\mathbb{R})=\mathbb{R}^{3}$ be a maximal Legendrian unknot. Then any two $L Q$ generating families of $L$ are equivalent.

Proof By Theorem 3.5, it suffices to prove the theorem in the case where $L$ is the unknot pictured in Figure 1. Let $f, g$ be two LQ generating families for $L$. By applying fiber-preserving diffeomorphisms and stabilizations, we can assume the following:

- $f$ and $g$ have the same domain $\mathbb{R} \times \mathbb{R} \times \mathbb{R}^{n}=\{(x, l, v)\}$; we will let $f_{x}, g_{x}: \mathbb{R} \times$ $\mathbb{R}^{n} \rightarrow \mathbb{R}$ denote the associated fiber functions.

- The critical points of $f_{x}$ agree with the critical points of $g_{x}$; we will let $C_{x} \subset$ $\mathbb{R} \times \mathbb{R}^{n}$ denote this set of critical points. Assuming that the two cusp points of $\pi_{x z}(L)$ occur at $(0,0)$ and $(1,0)$, we see that $C_{0}$ and $C_{1}$ consist of a single point with critical value $0, C_{x}$ for $x \in(0,1)$ consists of two nondegenerate critical points with nonzero critical values, and $C_{x}=\varnothing$ for all other $x$. By a Morse theoretic argument (see, for example, the proof of Proposition 4.3), for $x \in(0,1)$, we can write $C_{x}=\left\{v_{0}(x), v_{1}(x)\right\}$ where $v_{1}(x)$ has index $k+1$, $v_{0}(x)$ has index $k$, and

$$
f_{x}\left(v_{1}(x)\right)=g_{x}\left(v_{1}(x)\right)>0>f_{x}\left(v_{0}(x)\right)=g_{x}\left(v_{0}(x)\right) .
$$

- $f$ and $g$ are special; that is, outside a compact set of $\mathbb{R} \times \mathbb{R}^{n}$ they are strictly linear-quadratic, with linear and quadratic parts independent of the base point $x \in \mathbb{R}$.

We next show that for all $x$, we can assume $f_{x}=g_{x}$ on a neighborhood $U(x)$ of $C_{x}$ in $\mathbb{R} \times \mathbb{R}^{n}$. 
Lemma 3.7 There exists a neighborhood $U$ of $\cup_{x \in \mathbb{R}} C_{x}$ in $\mathbb{R} \times \mathbb{R} \times \mathbb{R}^{n}$ so that for $U(x)=U \cap\left(\{x\} \times \mathbb{R} \times \mathbb{R}^{n}\right)$, after fiber-preserving diffeomorphisms $f_{x}=g_{x}$ on $U(x)$, and all gradient trajectories of $f_{x}$ and of $g_{x}$ intersect $U(x)$ in a connected set.

Proof From Arnol'd, Guseĭn-Zade and Varchenko [2], we know that after applying fiber-preserving diffeomorphisms, in a neighborhood of $C_{0}$ in $\mathbb{R} \times \mathbb{R} \times \mathbb{R}^{n}$ we can assume $f(x, l, v)=g(x, l, v)=l^{3}-x l+Q(v)$, where $Q$ is a nondegenerate quadratic function. A similar statement holds in a neighborhood of $C_{1}$. Thus there is the desired neighborhood of $C_{0} \cup C_{1}$.

To see that there is a such a neighborhood for $\cup_{x \in \mathbb{B}} C_{x}$, we first note that with a generic choice of metrics, $f_{x}$ (and $\left.g_{x}\right), x \in(0,1)$, forms a family of functions whose gradient flows satisfy the Morse-Smale conditions. We will now argue that for all $x \in(0,1)$, there is a single isolated gradient trajectory of $f_{x}$ from $v_{1}(x)$ to $v_{0}(x)$. Since the gradient trajectories of $f_{x}$ satisfy the Morse-Smale conditions, for all $x \in(0,1)$, $W^{u}\left(v_{1}(x)\right)$ (the unstable manifold of $\left.v_{1}(x)\right), W^{s}\left(v_{0}(x)\right)$ (the stable manifold of $\left.v_{0}(x)\right)$, and $f_{x}^{-1}(0)$ intersect transversally in a finite number of points. Since near $x=0$, this intersection consists of a single point, for all $x$ this intersection consists of a single point. It follows that for all $x \in(0,1)$ there is a single gradient trajectory of $f_{x}$ in $W^{u}\left(v_{1}(x)\right) \cap W^{s}\left(v_{0}(x)\right)$. The analogous argument shows that there is a single gradient trajectory of $g_{x}$ from $v_{1}(x)$ to $v_{0}(x)$ for all $x \in(0,1)$.

It is not hard to show that by applying a diffeomorphism of $\mathbb{R}^{1+n}$, we can assume $f_{x}=g_{x}$ on neighborhoods $V_{0}(x), V_{1}(x)$ of $v_{0}(x)$ and $v_{1}(x)$, respectively. This can be proved using the Morse Lemma to obtain a diffeomorphism equating $f_{x}$ and $g_{x}$ on neighborhoods of $v_{0}$ and $v_{1}$ and then extending this diffeomorphism to all of $\mathbb{R}^{1+n}$ via the isotopy extension theorem using the fact that any embedding of two disjoint balls must be isotopic to the identity.

By applying this neighborhood diffeomorphism together with the fact that there is a single gradient trajectory from $v_{1}(x)$ to $v_{0}(x)$, after applying diffeomorphisms, we can assume that

- $v_{0}(x), v_{1}(x) \in \mathbb{R} \times\{0\}$ and $I(x):=W^{u}\left(v_{1}(x)\right) \cap W^{s}\left(v_{0}(x)\right) \subset \mathbb{R} \times\{0\} ;$

- For neighborhoods $V_{1}(x), V_{0}(x)$ of $v_{1}(x), v_{0}(x), f_{x}=g_{x}$ on $V_{1}(x) \cup$ $I(x) \cup V_{0}(x)$.

Now, there exist tubular neighborhoods $T_{f}(x)$ and $T_{g}(x)$ of the open interval $I(x)$ consisting of gradient trajectories of $f_{x}, g_{x}$ that intersect $V_{1}(x)$ and $V_{0}(x)$. Viewing each tubular neighborhood as a family of parameterized disks, we see that after applying 
a diffeomorphism, we can assume $T_{f}(x)=T_{g}(x)=T(x)$ and $f_{x}=g_{x}$ on $U(x)=$ $V_{0}(x) \cup T(x) \cup V_{1}(x)$.

The desired $U$ can be constructed from the above described neighborhoods of $C_{0}, C_{1}$, and $C_{x}, x \in \mathbb{R}$. This completes the proof of the lemma.

We will be using the gradient flows of $f_{x}$ and $g_{x}$ to define the diffeomorphism $\varphi_{x}$ of $\mathbb{R} \times \mathbb{R}^{n}$ such that $f_{x} \circ \varphi_{x}=g_{x}$. In particular, we will be working with the orbits of the gradient flows.

To become familiar with the construction, suppose we have a situation where every orbit of the gradient flow of both $f_{x}$ and $g_{x}$ intersect $U(x)$. In this case, our diffeomorphism $\varphi_{x}$ is defined by leaving all points in $U(x)$ fixed, while mapping points outside of $U(x)$ in the following way. For $w \notin U(x)$, flow along the orbit of $w$ by the positive (negative) gradient flow of $g_{x}$ until you reach a reference point $w^{\prime} \in U(x)$. Then flow from $w^{\prime}$ by the negative (positive) gradient flow of $f_{x}$ until you reach a point $w^{\prime \prime}$ such that $f_{x}\left(w^{\prime \prime}\right)=g_{x}(w)$ : since $f_{x}=g_{x}$ on $U(x)$, it is not difficult to verify that the $f_{x}$-orbit containing $w^{\prime}$ takes on the same values as the $g_{x}$-orbit containing $w^{\prime}$ and thus $w^{\prime \prime}$ must exist. Define $\varphi_{x}(w)=w^{\prime \prime}$, so then $f_{x} \circ \varphi_{x}=g_{x}$.

To see that this map is well-defined, suppose we choose a different reference point $\widetilde{w^{\prime}}$ in $U(x)$. Then $w^{\prime}$ and $\widetilde{w^{\prime}}$ are in the same orbit with respect to the gradient flow of $g_{x}$. Moreover since $w^{\prime}, \widetilde{w^{\prime}} \in U(x)$ and $f_{x}=g_{x}$ on $U(x)$, we also know that $w^{\prime}, \widetilde{w^{\prime}}$ are in the same orbit with respect to the gradient flow of $f_{x}$. Thus they both result in the same $w^{\prime \prime}$, so $\varphi_{x}$ is well-defined.

In practice, we will usually have to consider the case where not every orbit intersects $U(x)$. In this case, we will see that every orbit will intersect either $U(x)$ or a "negative infinity level set," where a negative infinity level set for $f$ (respectively for $g$ ) is defined to be $f_{x}^{-1}(c)$ (respectively $g_{x}^{-1}(c)$ ) for some fixed $c \ll 0$. Let $L_{-\infty}^{f}:=f_{x}^{-1}(c)$ and let $L_{-\infty}^{g}:=g_{x}^{-1}(c)$. Since $f$ and $g$ are assumed to be special, $L_{-\infty}^{f}$ and $L_{-\infty}^{g}$ do not depend on $x$. In fact, since $f$ is linear-quadratic at infinity,

$$
\begin{aligned}
L_{-\infty}^{f} & =\left\{\left(l, v_{1}, \ldots, v_{n}\right) \in \mathbb{R}^{1+n} \mid l-v_{1}^{2}-\cdots-v_{j}^{2}+v_{j+1}^{2}+\cdots+v_{n}^{2}=c\right\} \\
& =\left\{\left(c+v_{1}^{2}+\cdots+v_{j}^{2}-v_{j+1}^{2}-\cdots-v_{n}^{2}, v_{1}, v_{2}, \ldots, v_{n}\right) \in \mathbb{R}^{1+n}\right\} .
\end{aligned}
$$

Therefore $L_{-\infty}^{f}$ is an embedded image of $\mathbb{R}^{n}$ in $\mathbb{R} \times \mathbb{R}^{n}$, as is $L_{-\infty}^{g}$. To see that each orbit of the gradient flows of $f_{x}\left(g_{x}\right)$ intersects $U(x)$ or $L_{-\infty}^{f}\left(L_{-\infty}^{g}\right)$, observe that an orbit will either terminate at a critical point or enter the region of $\mathbb{R} \times \mathbb{R}^{n}$ where $f_{x}$ $\left(g_{x}\right)$ is standard linear-quadratic function. In the first case the orbit intersects $U(x)$, and in the second case the orbit intersects $L_{-\infty}^{f}\left(L_{-\infty}^{g}\right)$. 
The idea now is to define a diffeomorphism similar to the one above, but using reference points in $U(x) \cup L_{-\infty}^{f}$ and $U(x) \cup L_{-\infty}^{g}$. However we must be careful to be sure that the map is well-defined with respect to the reference point chosen. The difficulty here is when we have orbits of the gradient flows of $f_{x}\left(g_{x}\right)$ that intersect both $U(x)$ and $L_{-\infty}^{f}\left(L_{-\infty}^{g}\right)$, and thus we have reference points in both $U(x)$ and $L_{-\infty}^{f}\left(L_{-\infty}^{g}\right)$. We will now show that the map is, in fact, well defined.

Shrink $U(x)$ slightly to a closed set $D(x) \subset U(x)$ that satisfies the conditions on $U(x)$ specified by Lemma 3.7. Consider the set of orbits that intersect both $D(x) \subset U(x)$ and $L_{-\infty}^{f}\left(\right.$ or $\left.L_{-\infty}^{g}\right)$. Let $W^{f}(x)\left(W^{g}(x)\right)$ denote a transverse slice of these orbits so that each orbit with respect to the gradient flow of $f_{x}\left(g_{x}\right)$ intersects $W^{f}(x)$ ( $\left.W^{g}(x)\right)$ precisely once. In fact, $W^{f}(x)\left(W^{g}(x)\right)$ can be chosen to be closed $n$-dimensional disks. Since $f_{x}=g_{x}$ on $D(x) \subset U(x)$, it is possible to choose $W(x):=W^{f}(x)=W^{g}(x) \subset D(x) \subset U(x)$. For $w \in W(x)$, let $w_{g}, w_{f}$ be the orbits of $w$ with respect to the gradient flows of $g_{x}, f_{x}$ respectively. Note $w_{g} \cap L_{-\infty}^{g}$ and $w_{f} \cap L_{-\infty}^{f}$ are each single points, call them $w_{g}^{-\infty}$ and $w_{f}^{-\infty}$ respectively. Let $W_{1}(x)=\cup_{w \in W(x)} w_{g}^{-\infty}, W_{2}(x)=\cup_{w \in W(x)} w_{f}^{-\infty}$, and consider $\theta_{x}: W_{1}(x) \rightarrow W_{2}(x)$ defined by $\theta_{x}\left(w_{g}^{-\infty}\right)=w_{f}^{-\infty}$ for each $w \in W(x)$. Then by an application of the isotopy extension theorem, $\theta_{x}$ extends to a diffeomorphism $\Theta_{x}: L_{-\infty}^{g} \rightarrow L_{-\infty}^{f}$.

Now we proceed to define $\varphi_{x}$. As before, $\varphi_{x}$ leaves all points in $D(x) \subset U(x)$ fixed. For $w \notin D(x)$, flow along the orbit of $w$ by the positive (negative) gradient flow of $g_{x}$ until you reach a reference point $w^{\prime} \in D(x) \cup L_{-\infty}^{g}$. If $w^{\prime} \in D(x)$, let $w^{\prime \prime}=w^{\prime}$. If $w^{\prime} \in L_{-\infty}^{g}$, let $w^{\prime \prime}=\Theta_{x}\left(w^{\prime}\right)$. Now flow from $w^{\prime \prime}$ by the negative (positive) gradient flow of $f_{x}$ until you reach a point $w^{\prime \prime \prime}$ such that $f_{x}\left(w^{\prime \prime \prime}\right)=g_{x}(w)$. Define $\varphi_{x}(w)=w^{\prime \prime \prime}$, so that $f_{x} \circ \varphi_{x}=g_{x}$. It is straight forward to verify that $\varphi_{x}$ is well-defined.

Now for each $x \in \mathbb{R}$ we have a diffeomorphism $\varphi_{x}$ of $\mathbb{R} \times \mathbb{R}^{n}$ such that $f_{x} \circ \varphi_{x}=g_{x}$. By construction, $\varphi_{x}$ varies smoothly with $x$. Thus we have a diffeomorphism $\Phi$ of $\mathbb{R} \times \mathbb{R} \times \mathbb{R}^{n}$ such that $f \circ \Phi=g$. Hence $f$ and $g$ are equivalent.

Remark 3.8 The above proof and Théret's proof of uniqueness of generating families for the Lagrangian 0 -section have some differences. In Théret's setting, the construction of the set $U(x)$ containing the fiber-critical point where the function is standard is an easy consequence of Morse Theory. Also, in Théret's work, he uses a $-\epsilon-$ level set where above we use a $-\infty$-level set. When using the $-\epsilon-$ level set, it is more immediate that the fiber diffeomorphisms constructed via gradient flows are well defined. However, Théret must spend a great deal of effort to prove that for two generating families $f$ and $g$, there is a global diffeomorphism between $f^{-1}(-\epsilon)$ and $g^{-1}(-\epsilon)$ that is a 
diffeomorphism on each fiber slice. This difficulty is avoided in the above proof with the use of $-\infty$-level sets.

\section{LQ generating family polynomials}

We now use the existence and uniqueness results of the previous section to define invariant polynomials for two component Legendrian links in $\mathbb{R}^{3}$ where each component is a maximal unknot. Much of this material parallels the results from Traynor [19]; however, in that paper links live in $\mathcal{J}^{1}\left(S^{1}\right)$ and each component of the link was Legendrian isotopic to the 1 -jet of a function.

Definition 4.1 Given two functions $f_{i}: \mathbb{R} \times \mathbb{R}^{1+n_{i}} \rightarrow \mathbb{R}, i=0,1$, let $\Delta: \mathbb{R} \times \mathbb{R}^{1+n_{1}} \times$ $\mathbb{R}^{1+n_{0}} \rightarrow \mathbb{R}$ be given by $\Delta\left(x, l_{1}, v_{1}, l_{0}, v_{0}\right)=f_{1}\left(x, l_{1}, v_{1}\right)-f_{0}\left(x, l_{0}, v_{0}\right)$. Then $\Delta$ is the difference function of $f_{1}$ and $f_{0}$. Furthermore, if $f_{1}$ and $f_{0}$ generate $\Lambda_{1}$ and $\Lambda_{0}$, respectively, then $\Delta$ is a difference function of $L=\left(\Lambda_{1}, \Lambda_{0}\right)$.

Note the following facts about the critical points of a difference function $\Delta$. First, if $\Delta$ is a difference function for a link $L=\left(\Lambda_{1}, \Lambda_{0}\right)$, then the critical points of $\Delta$ are in oneto-one correspondence with points of the form $\left(\left(x_{0}, y_{0}, z_{1}\right),\left(x_{0}, y_{0}, z_{0}\right)\right) \in \Lambda_{1} \times \Lambda_{0}$. This can be seen by calculating the derivative of $\Delta$ in terms of the derivatives of $f_{1}$ and $f_{0}$, where $f_{i}$ generates $\Lambda_{i}(i=0,1)$. Therefore a critical point of $\Delta$ can be identified in the front projection of $L$ as a pair of points, one point on each $\Lambda_{i}$, where the points have the same $x$ coordinate and the same slope. Second, if $L=\left(\Lambda_{1}, \Lambda_{0}\right)$ is a link, the components of $L$ do not intersect, and thus 0 is not a critical value of $\Delta$.

We will now proceed to define homology groups for $\Delta$, where $\Delta: \mathbb{R} \times \mathbb{R}^{1+n_{1}} \times \mathbb{R}^{1+n_{0}} \rightarrow$ $\mathbb{R}$ is a difference function for $L$. For $c \in \mathbb{R}, c$ a noncritical value of $\Delta$, let

$$
\Delta^{c}:=\left\{\left(x, l_{1}, v_{1}, l_{0}, v_{0}\right) \mid \Delta\left(x, l_{1}, v_{1}, l_{0}, v_{0}\right) \leq c\right\} .
$$

Note that for every link $L$ and difference function $\Delta$, there exists some constant $m>0$ such that all the critical values of $\Delta$ are in the interval $[-m+\epsilon, m-\epsilon]$ for some $\epsilon>0$. Then we define

$$
\Delta^{\infty}:=\Delta^{m}, \quad \Delta^{-\infty}:=\Delta^{-m} .
$$

Now the homology groups for $\Delta$ are defined as follows.

Definition 4.2 Let $\Delta$ be a difference function for a Legendrian link $L=\left(\Lambda_{1}, \Lambda_{0}\right)$. The total, positive, and negative homology groups of $L$ with respect to $\Delta$ are defined 
as

$$
\begin{aligned}
H_{k}(\Delta) & =H_{k}\left(\Delta^{\infty}, \Delta^{-\infty}\right), \\
H_{k}^{+}(\Delta) & =H_{k}\left(\Delta^{\infty}, \Delta^{0}\right), \\
H_{k}^{-}(\Delta) & =H_{k}\left(\Delta^{0}, \Delta^{-\infty}\right),
\end{aligned}
$$

for $k \in \mathbb{Z}$, where the relative homology groups are calculated with coefficients in $\mathbb{Z} / 2 \mathbb{Z}$.

If $\Delta$ and $\Delta^{\prime}$ are two difference functions for a link $L$, then their homology groups will be related as follows. If $\Delta$ and $\Delta^{\prime}$ differ only by a fiber-preserving diffeomorphism, then they will have the same homology groups. However if $\Delta$ and $\Delta^{\prime}$ differ by a stabilization, then there exists $i \in \mathbb{Z}$ such that

$$
H_{k+i}(\Delta)=H_{k}\left(\Delta^{\prime}\right), \quad H_{k+i}^{+}(\Delta)=H_{k}^{+}\left(\Delta^{\prime}\right), \quad H_{k+i}^{-}(\Delta)=H_{k}^{-}\left(\Delta^{\prime}\right) .
$$

In fact, the total homology groups do not carry any information about a particular link.

Proposition 4.3 Let $L=\left(\Lambda_{1}, \Lambda_{0}\right)$ be a Legendrian link of maximal unknots. Given a difference function $\Delta$ for $L, H_{k}(\Delta) \simeq 0$ for all $k \in \mathbb{Z}$.

To prove this proposition, we will use the following lemma. The lemma is similar to [19, Lemma 3.10], and can be proved following the argument presented there.

Lemma 4.4 Consider a smooth 1-parameter family of difference functions $\Delta_{t}: \mathbb{R} \times$ $\mathbb{R}^{1+n_{1}} \times \mathbb{R}^{1+n_{0}} \rightarrow \mathbb{R}, t \in[0,1]$, where each $\Delta_{t}$ is the difference of two LQ generating families. Given paths $\alpha, \beta:[0,1] \rightarrow \mathbb{R}$ such that, for all $t, \alpha(t), \beta(t)$ are noncritical values of $\Delta_{t}$ with $\alpha(t)<\beta(t)$. Then for any $t \in[0,1]$ and $k \in \mathbb{Z}, H_{k}\left(\Delta_{0}^{\beta(0)}, \Delta_{0}^{\alpha(0)}\right) \simeq$ $H_{k}\left(\Delta_{t}^{\beta(t)}, \Delta_{t}^{\alpha(t)}\right)$.

Using this, we now prove Proposition 4.3:

Proof Recall that if $\Delta$ and $\Delta^{\prime}$ are two difference functions for $L$, then there exists some $i \in \mathbb{Z}$ such that $H_{k+i}(\Delta) \simeq H_{k}\left(\Delta^{\prime}\right)$. Therefore if the theorem is true for one particular difference function of $L$, then it is true for every difference function.

By hypothesis, each strand of $L$ can be individually isotoped so that it is the maximal unknot shown in Figure 1. Choose isotopies of the strands $\Lambda_{1}^{t}, \Lambda_{0}^{t}, t \in[0,1]$, so that $\Lambda_{1}^{0}=\Lambda_{1}, \Lambda_{0}^{0}=\Lambda_{0}$, and when $t=1, \Lambda_{1}^{1}$ and $\Lambda_{0}^{1}$ are maximal unknots translated so that $\left(x_{1}, y_{1}, z_{1}\right) \in \Lambda_{1}^{1}$ and $\left(x_{0}, y_{0}, z_{0}\right) \in \Lambda_{0}^{1}$ implies that $x_{1} \neq x_{0}$; in other words, $\Lambda_{1}^{1}$ and $\Lambda_{0}^{1}$ have no base points in $\mathbb{R}$ in common. Consider $L_{t}=\left(\Lambda_{1}^{t}, \Lambda_{0}^{t}\right), t \in[0,1]$. If 
$L$ is a nontrivial link, this is not a link isotopy, but at each $t$, there will be a difference function, $\Delta_{t}$, for $L_{t}$ (which may have 0 as a critical value). Since critical points of $\Delta_{t}$ correspond to points $\left(\left(x_{0}, y_{0}, z_{1}\right),\left(x_{0}, y_{0}, z_{0}\right)\right) \in \Lambda_{1}^{t} \times \Lambda_{0}^{t}, \Delta_{1}$ has no critical points at all. Therefore $H_{k}\left(\Delta_{1}\right) \simeq 0$ for all $k \in \mathbb{Z}$. It remains to show that $H_{k}\left(\Delta_{0}\right) \simeq H_{k}\left(\Delta_{1}\right)$. For the family of difference functions $\Delta_{t}$, choose paths $\alpha, \beta:[0,1] \rightarrow \mathbb{R}$ such that $\alpha(t)$ is negative and less than all critical values of $\Delta_{t}$ and $\beta(t)$ is positive and greater than all critical values of $\Delta_{t}$. By Lemma 4.4, $H_{k}\left(\Delta_{0}\right) \simeq H_{k}\left(\Delta_{1}\right)$ for all $k \in \mathbb{Z}$. Therefore $H_{k}\left(\Delta_{0}\right) \simeq 0$ for all $k \in \mathbb{Z}$.

As we will see, the positive and negative homology groups do carry information about a particular link. We will use polynomials $\Gamma_{\Delta}^{ \pm}$to encode information about the set of homology groups of a difference function $\Delta$. Define

$$
\begin{aligned}
& \Gamma_{\Delta}^{+}(\lambda)=\sum_{k=0}^{\infty} \operatorname{dim} H_{k}^{+}(\Delta) \cdot \lambda^{k}, \\
& \Gamma_{\Delta}^{-}(\lambda)=\sum_{k=0}^{\infty} \operatorname{dim} H_{k}^{-}(\Delta) \cdot \lambda^{k} .
\end{aligned}
$$

As noted above, if $\Delta$ and $\Delta^{\prime}$ are two difference functions for a link $L$, then there exists some $i \in \mathbb{Z}$ such that $\Gamma_{\Delta}^{ \pm}(\lambda)=\Gamma_{\Delta^{\prime}}^{ \pm}(\lambda) \cdot \lambda^{i}$. Thus we define positive and negative homology polynomials for $L$ as normalized versions of the positive and negative homology polynomials for $\Delta$, where $\Delta$ is some difference function for $L$.

Definition 4.5 Let $L=\left(\Lambda_{1}, \Lambda_{0}\right)$ be a Legendrian link of maximal unknots with difference function $\Delta$. Define the positive and negative homology polynomials of $L$ by

$$
\begin{aligned}
& \Gamma^{+}(\lambda)[L]=\Gamma_{\Delta}^{+}(\lambda) \cdot \lambda^{i}, \\
& \Gamma^{-}(\lambda)[L]=\Gamma_{\Delta}^{-}(\lambda) \cdot \lambda^{i},
\end{aligned}
$$

where $i \in \mathbb{Z}$ is chosen so that $\Gamma^{-}(\lambda)[L]$ has degree zero.

Remark 4.6 An alternative to looking at a normalized version of the polynomials is to consider a vector encoding the dimensions of the homology groups. For example, if $\operatorname{dim} H_{k}^{-}(\Delta)=0$ if $k<A$ or $k>B$ and $H_{A}^{-}(\Delta)$ and $H_{B}^{-}(\Delta)$ are nontrivial, then we construct the negative homology vector

$$
\left(\operatorname{dim} H_{A}^{-}(\Delta), \operatorname{dim} H_{A+1}^{-}(\Delta), \ldots, \operatorname{dim} H_{B}^{-}(\Delta)\right) .
$$

Similarly, one can construct the positive homology vector. 
In fact, the homology polynomials are invariants of $L$.

Theorem 4.7 $\Gamma^{ \pm}(\lambda)[L]$ are well-defined invariants of a Legendrian link $L=$ $\left(\Lambda_{1}, \Lambda_{0}\right)$ of maximal unknots.

Proof We have already seen that the homology polynomials for $L$ do not depend on the choice of difference function. It remains to show that the polynomials do not change as $L$ undergoes a Legendrian isotopy.

Suppose $L_{t}, t \in[0,1]$, is a 1-parameter family of Legendrian links made of two maximal unknots. By Theorem 3.1, there exists a difference function $\Delta_{t}$ for each $L_{t}$ such that $\Delta_{0}=\Delta_{t}$ outside a compact set. We will show that for each $t \in[0,1]$, we have

$$
H_{k}^{+}\left(\Delta_{t}\right) \simeq H_{k}^{+}\left(\Delta_{0}\right), \quad H_{k}^{-}\left(\Delta_{t}\right) \simeq H_{k}^{-}\left(\Delta_{0}\right),
$$

for all $k \in \mathbb{Z}$. Choose paths $\alpha, \beta, \gamma:[0,1] \rightarrow \mathbb{R}$ such that $\alpha(t)$ is negative and is less than all critical values of $\Delta_{t}, \beta(t) \equiv 0$, and $\gamma(t)$ is positive and is greater than all critical values of $\Delta_{t}$. Then by construction (and since 0 is never a critical value of $\left.\Delta_{t}\right), \alpha(t), \beta(t)$, and $\gamma(t)$ are noncritical values of $\Delta_{t}$ for all $t$. Thus by Lemma 4.4, the above result holds.

In the remainder of this section, we will prove a few facts about the homology groups and homology polynomials for a Legendrian link made of two maximal unknots.

The following lemma will be used to relate the positive and negative homology polynomials for $L$ and to calculate polynomials for particular links in Section 5. This Lemma agrees with [19, Lemma 3.13] and the proof can be found there.

Lemma 4.8 For a function $\Delta: \mathbb{R} \times \mathbb{R}^{1+n_{1}} \times \mathbb{R}^{1+n_{0}} \rightarrow \mathbb{R}$, and $a, b, c$ noncritical values of $\Delta$ with $a<b<c$, there is a long exact sequence

$\cdots \stackrel{\partial_{*}}{\longrightarrow} H_{k}\left(\Delta^{b}, \Delta^{a}\right) \stackrel{i_{*}}{\longrightarrow} H_{k}\left(\Delta^{c}, \Delta^{a}\right) \stackrel{\pi_{*}}{\longrightarrow} H_{k}\left(\Delta^{c}, \Delta^{b}\right) \stackrel{\partial_{*}}{\longrightarrow} H_{k-1}\left(\Delta^{b}, \Delta^{a}\right) \stackrel{i_{*}}{\longrightarrow} \cdots$.

Theorem 4.9 Let $L=\left(\Lambda_{1}, \Lambda_{0}\right)$ be a Legendrian link where $\Lambda_{1}$ and $\Lambda_{0}$ are maximal unknots. Then $\Gamma^{+}(\lambda)[L]=\lambda \cdot \Gamma^{-}(\lambda)[L]$.

Remark 4.10 Notice that in the vector notation described in Remark 4.6, this says that our links will have the same negative and positive homology vectors. Because of this dependence of $\Gamma^{+}(\lambda)[L]$ on $\Gamma^{-}(\lambda)[L]$, in the following result statements, usually only $\Gamma^{-}(\lambda)[L]$ will be discussed. 
Proof Let $\Delta$ be a difference function for $L$. Then by Proposition $4.3, H_{k}(\Delta) \simeq 0$ for all $k \in \mathbb{Z}$. Choose $m \in \mathbb{R}$ large enough so that all the critical values of $\Delta$ are within the interval $[-m+\epsilon, m-\epsilon]$ for some $\epsilon>0$, and recall that 0 is a noncritical value of $\Delta$. Therefore by Lemma 4.8 we have the following exact sequence, for any $k \in \mathbb{Z}$.

$$
\cdots \stackrel{i_{*}}{\longrightarrow} H_{k}\left(\Delta^{m}, \Delta^{-m}\right) \stackrel{\pi_{*}}{\longrightarrow} H_{k}\left(\Delta^{m}, \Delta^{0}\right) \stackrel{\partial_{*}}{\longrightarrow} H_{k-1}\left(\Delta^{0}, \Delta^{-m}\right) \stackrel{i_{*}}{\longrightarrow} \cdots .
$$

Note that

$$
\begin{aligned}
H_{k}\left(\Delta^{m}, \Delta^{-m}\right) & \simeq H_{k}(\Delta) \simeq 0, \\
H_{k}\left(\Delta^{m}, \Delta^{0}\right) & \simeq H_{k}^{+}(\Delta), \\
\text { and } \quad H_{k}\left(\Delta^{0}, \Delta^{-m}\right) & \simeq H_{k}^{-}(\Delta) .
\end{aligned}
$$

Thus the above sequence can be rewritten as

$$
\cdots \stackrel{i_{*}}{\longrightarrow} 0 \stackrel{\pi_{*}}{\longrightarrow} H_{k}^{+}(\Delta) \stackrel{\partial_{*}}{\longrightarrow} H_{k-1}^{-}(\Delta) \stackrel{i_{*}}{\longrightarrow} 0 \stackrel{\pi_{*}}{\longrightarrow} \cdots .
$$

Hence the $\partial_{*}$ maps are all isomorphisms, which tells us that for all $k \in \mathbb{Z}, H_{k+1}^{+}(\Delta) \simeq$ $H_{k}^{-}(\Delta)$.

Finally, we will end the section by showing how the negative homology polynomials can sometimes detect if a link is "ordered". We say a link $L=\left(\Lambda_{1}, \Lambda_{0}\right)$ is ordered if it is not Legendrian equivalent to $\bar{L}=\left(\Lambda_{0}, \Lambda_{1}\right)$.

Theorem 4.11 Let $L=\left(\Lambda_{1}, \Lambda_{0}\right)$ be a Legendrian link of maximal unknots. If there does not exist an $l \in \mathbb{Z}$ such that $\Gamma^{-}(\lambda)[L]=\lambda^{l} \cdot \Gamma^{-}\left(\lambda^{-1}\right)[L]$, then the link $L$ is ordered.

Remark 4.12 In terms of the vector notation, the above corollary says that a link $L$ is ordered if the vector associated to $\Gamma^{-}$is not symmetric. For example, it will be shown that the rational link $L=(2,1,4)$, which is shown on the left side of Figure 6 , has $\Gamma^{-}(\lambda)[L]=2 \lambda^{0}+\lambda^{-1}$ and so a corresponding vector of $(1,2)$, and therefore it must be ordered. However the rational link $L^{\prime}=(2,1,2)$, with $\Gamma^{-}(\lambda)\left[L^{\prime}\right]=\lambda^{0}+\lambda^{-1}$ or vector equal to $(1,1)$ is potentially unordered.

Proof Let $L=\left(\Lambda_{1}, \Lambda_{0}\right), \bar{L}=\left(\Lambda_{0}, \Lambda_{1}\right)$. We will show that there exists an integer $l \in \mathbb{Z}$ such that $\Gamma^{-}(\lambda)[L]=\lambda^{l} \cdot \Gamma^{-}\left(\lambda^{-1}\right)[\bar{L}]$. Let $F_{1}, F_{0}$ be LQ generating families for $\Lambda_{1}$ and $\Lambda_{0}$, respectively. Then $\Delta=F_{1}-F_{0}$ is a difference function for $L$ and $\bar{\Delta}=F_{0}-F_{1}=-\Delta$ is a difference function for $\bar{L}$. Then if $N$ denotes the dimension 
of the domain of $\Delta$, we have

$$
\begin{aligned}
H_{k}^{+}(\Delta)=H_{k}\left(\Delta^{+\infty}, \Delta^{0}\right) & \simeq H^{N-k}\left(\Delta^{+\infty}, \Delta^{0}\right) \\
& \simeq H_{N-k}\left(\bar{\Delta}^{0}, \bar{\Delta}^{-\infty}\right)=H_{N-k}^{-}(\bar{\Delta}) .
\end{aligned}
$$

Therefore the homology polynomials of $\Delta$ and the homology polynomials of $\bar{\Delta}$ are related as follows:

$$
\Gamma_{\Delta}^{-}(\lambda)=\lambda^{N} \cdot \Gamma_{\frac{\Delta}{\Delta}}^{+}\left(\lambda^{-1}\right)
$$

Since the homology polynomials of $L$ and $\bar{L}$ are normalized versions of the polynomials of $\Delta$ and $\bar{\Delta}$, there exists an integer $l^{\prime}$ such that

$$
\Gamma^{-}(\lambda)[L]=\lambda^{l^{\prime}} \cdot \Gamma^{+}\left(\lambda^{-1}\right)[\bar{L}] \text {. }
$$

By Theorem 4.9, $\Gamma^{+}\left(\lambda^{-1}\right)[\bar{L}]=\lambda^{-1} \cdot \Gamma^{-}\left(\lambda^{-1}\right)[L]$. Therefore

$$
\Gamma^{-}(\lambda)[L]=\lambda^{l^{\prime}} \cdot \Gamma^{+}\left(\lambda^{-1}\right)[\bar{L}]=\lambda^{l^{\prime}} \cdot \lambda^{-1} \cdot \Gamma^{-}\left(\lambda^{-1}\right)[\bar{L}] .
$$

\section{Polynomial calculations}

In order to calculate the homology polynomials for a given Legendrian link $L=$ $\left(\Lambda_{1}, \Lambda_{0}\right)$, it will sometimes be sufficient to know the nondegenerate critical points of a difference function for $L$, as well as the critical values and indices of the critical points. Recall that a critical point corresponds to a point $\left(\left(x_{0}, y_{0}, z_{1}\right),\left(x_{0}, y_{0}, z_{0}\right)\right) \in \Lambda_{1} \times \Lambda_{0}$, and the critical value is equal to $z_{1}-z_{0}$. Thus we can find all critical points and their values from looking at the front projection of $L$, without finding the difference function $\Delta$ explicitly. In this section we explain how we can also recover the index of a critical point, up to a shift, from the front projection of $L$. The definitions and propositions in this section are based on those found in [19, Section 5].

We begin with some definitions for use with the front projection of a knot or link. Given a Legendrian knot $\Lambda \subset \mathcal{J}^{1}(\mathbb{R})$, let $\pi_{x z}(\Lambda)$ be the front projection of $\Lambda$. Let $C$ be the set of points in $\Lambda$ whose image under $\pi_{x z}$ is a cusp point. We define the branches of $\Lambda$ to be the connected components of $\Lambda \backslash C$. Branches $B_{0}, B_{1}$ are said to be adjacent if their closures, $\overline{B_{0}}$ and $\overline{B_{1}}$, intersect. Given two adjacent branches $B_{0}$ and $B_{1}$, we say $B_{1}>B_{0}$ if there exists some $b \in \overline{B_{0}} \cap \overline{B_{1}}$ and a path $\gamma:[0,1] \rightarrow \pi_{x z}(\Lambda)$ such that $\gamma\left[0, \frac{1}{2}\right) \subset \pi_{x z}\left(B_{0}\right), \gamma\left(\frac{1}{2}, 0\right] \subset \pi_{x z}\left(B_{1}\right)$, and $\gamma\left(\frac{1}{2}\right)=\pi_{x z}(b)$ where $\pi_{x z}(b)$ is an up-cusp along the path.

Now we will describe a way of assigning integers, called branch indices, to each branch of a knot. Let $\Lambda \subset \mathcal{J}^{1}(\mathbb{R})$ be a maximal unknot, and choose $p_{0}$ to be a marked point of 
$\Lambda$ such that $\pi_{x z}\left(p_{0}\right)$ is not a cusp point of $\pi_{x z}(\Lambda)$. Let $\left\{B_{i}\right\}$ be the set of branches of $\Lambda$ such that $B_{0}$ is the branch containing $p_{0}$. We say $B_{0}$ is the initial branch of $\Lambda$. We then define the branch index $i_{B r}:\left\{B_{i}\right\} \rightarrow \mathbb{Z}$ as follows:

(1) $i_{B r}\left(B_{0}\right)=0$, and

(2) $i_{B r}\left(B_{i}\right)-i_{B r}\left(B_{j}\right)=1$ if $B_{i}, B_{j}$ are adjacent with $B_{i}>B_{j}$.

Note that given a marked point, the branch index is well-defined for all branches of $\Lambda$. Recall that for each point on a branch, there is a corresponding critical point of the generating family restricted to a fiber. As explained in [19, Proposition 5.3], there is some integer so that the branch index of a point corresponds, up to a shift by this integer, to the index of the corresponding fiber critical point.

The final piece we need to calculate indices of critical points of a difference function $\Delta$ is called the graph index of a critical point.

Definition 5.1 Let $\Lambda_{1}, \Lambda_{0} \subset \mathcal{J}^{1}(\mathbb{R})$ be Legendrian knots with branches $B_{1}, B_{0}$ with $\left(x_{0}, y_{0}, z_{1}\right) \in B_{1}$ and $\left(x_{0}, y_{0}, z_{0}\right) \in B_{0}$. Then there exists a neighborhood $U$ of $x_{0}$ in $\mathbb{R}$ and functions $g_{1}, g_{0}: U \rightarrow \mathbb{R}$ such that near $\left(x_{0}, y_{0}, z_{1}\right), \pi_{x z}\left(B_{1}\right)=\left\{\left(x, g_{1}(x)\right)\right\}$ and near $\left(x_{0}, y_{0}, z_{0}\right), \pi_{x z}\left(B_{0}\right)=\left\{\left(x, g_{0}(x)\right)\right\}$. Note that $x_{0}$ is a critical point of $\Gamma=g_{1}-g_{0}$. We say $\left(\left(x_{0}, y_{0}, z_{1}\right),\left(x_{0}, y_{0}, z_{0}\right)\right) \in \Lambda_{1} \times \Lambda_{0}$ is nondegenerate if $x_{0}$ is a nondegenerate critical point of $g_{1}-g_{0}$. The graph index $i_{\Gamma}$ of a nondegenerate critical point $x_{0}$ is the Morse index of $g_{1}-g_{0}$ at $x_{0}$.

The following proposition allows us to calculate the indices of critical points of a difference function $\Delta$.

Proposition 5.2 Let $L=\left(\Lambda_{1}, \Lambda_{0}\right)$ be a Legendrian link of maximal unknots, and let $\Delta$ be a difference function for $L$. Suppose $q \in \mathbb{R} \times \mathbb{R}^{1+n_{1}} \times \mathbb{R}^{1+n_{0}}$ is a nondegenerate critical point of $\Delta$ with corresponding point $\left(\left(x_{0}, y_{0}, z_{1}\right),\left(x_{0}, y_{0}, z_{0}\right)\right) \in \Lambda_{1} \times \Lambda_{0}$. Say that $B_{1}, B_{0}$ are the branches of $\Lambda_{1}, \Lambda_{0}$ containing $\left(x_{0}, y_{0}, z_{1}\right),\left(x_{0}, y_{0}, z_{0}\right)$, respectively. Then, for any choice of initial branches for $\Lambda_{1}$ and $\Lambda_{0}$, there is a $c \in \mathbb{Z}$ such that the index of $q$ is equal to $i_{B r}\left(B_{1}\right)-i_{B r}\left(B_{0}\right)+i_{\Gamma}\left(x_{0}\right)+c$.

We will often refer to the integer $i_{B r}\left(B_{1}\right)-i_{B r}\left(B_{0}\right)+i_{\Gamma}\left(x_{0}\right)$ as the relative index of $q$; this relative index is only well-defined up to a constant depending on the choice of initial branches. Proposition 5.2 can be proved with minor adaptions to the proof of [19, Proposition 5.5].

The following lemma will be our main tool for calculating the homology polynomials of particular links. This is essentially [19, Proposition 4.2]. The proof found there consists of studying a number of long exact sequences as given by Lemma 4.8. 
Lemma 5.3 Suppose the Legendrian link $L=\left(\Lambda_{1}, \Lambda_{0}\right)$ of maximal unknots has a difference function $\Delta: \mathbb{R} \times \mathbb{R}^{1+n_{1}} \times \mathbb{R}^{1+n_{0}} \rightarrow \mathbb{R}$ with critical values $c_{0}^{ \pm}, c_{1}^{ \pm}, \ldots, c_{n}^{ \pm}$ and noncritical values $a_{0}, a_{1}, \ldots, a_{n}, b_{0}, b_{1}, \ldots, b_{n}$ satisfying

$$
a_{0}<c_{0}^{-}<a_{1}<c_{1}^{-}<\cdots<a_{n}<c_{n}^{-}<0<c_{n}^{+}<b_{n}<\cdots<c_{1}^{+}<b_{1}<c_{0}^{+}<b_{0} .
$$

If

(1) For $j=0,1, \ldots, n$, there exist $w_{j}$ nondegenerate critical points with value $c_{j}^{+}$ and $w_{j}$ nondegenerate critical points with value $c_{j}^{-}$;

(2) For a given labeling of branch indices, all critical points of value $c_{j}^{+}$have relative index $i_{j}+1$ and all critical points of value $c_{j}^{-}$have relative index $i_{j}$;

(3) For $j=1,2, \ldots, n, H_{*}\left(\Delta^{b_{j}}, \Delta^{a_{j}}\right)=0$ for all $* \in \mathbb{Z}$;

Then

$$
\Gamma^{-}(\lambda)[L]=\lambda^{h} \cdot \sum_{j=0}^{n} w_{j} \lambda^{i_{j}},
$$

where $h$ is chosen so that $\Gamma^{-}(\lambda)[L]$ has degree 0 .

We can now state and prove formulas for the homology polynomials for several types of links. The first links we consider are the rational links $\left(2 w_{n}, k_{n}, 2 w_{n-1}, \ldots, k_{1}, 2 w_{0}\right)$ as described in Section 1. Theorem 5.4 is similar to [19, Theorem 6.1], and the proof follows the same format.

Theorem 5.4 Let $L=\left(2 w_{n}, k_{n}, 2 w_{n-1}, \ldots, k_{1}, 2 w_{0}\right)$ (see Figure 9). Then

$$
\Gamma^{-}(\lambda)[L]=w_{0} \lambda^{0}+w_{1} \lambda^{-k_{1}}+w_{2} \lambda^{-\left(k_{1}+k_{2}\right)}+\cdots+w_{n} \lambda^{-\left(k_{1}+\cdots+k_{n}\right)} .
$$

Remark 5.5 Using the vector notation, this says that the negative homology polynomial of $L=\left(2 w_{n}, k_{n}, 2 w_{n-1}, \ldots, k_{1}, 2 w_{0}\right)$ is given by the vector

$$
\left(w_{n}, 0, \ldots, 0, w_{n-1}, 0, \ldots, 0, w_{0}\right)
$$

where there are precisely $\left(k_{j}-1\right)$ zeros between $w_{j}$ and $w_{j-1}$.

Proof It is possible to isotope $L$ so that it has a difference function $\Delta: \mathbb{R} \times \mathbb{R}^{1+n_{1}} \times$ $\mathbb{R}^{1+n_{0}} \rightarrow \mathbb{R}$ with $2\left(w_{0}+w_{1}+\cdots+w_{n}\right)$ nondegenerate critical points. In particular, we can choose the branch indices such that, for $i=0,1, \ldots, n$, we have the following: $\Delta$ has $w_{i}$ critical points with critical value $c_{i}^{+}>0$ and relative index 1 if $i=0$, or relative index $1-\sum_{j=1}^{i} k_{j}$ otherwise, and $w_{i}$ critical points with critical value $c_{i}^{-}<0$ 


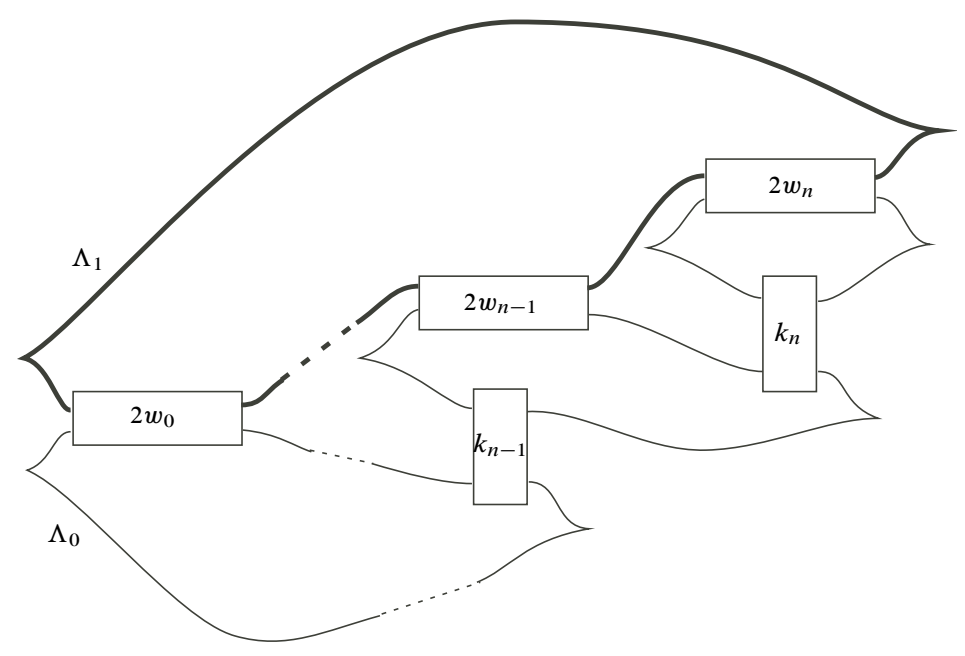

Figure 9: The link $L=\left(2 w_{n}, k_{n}, \ldots, 2 w_{1}, k_{1}, 2 w_{0}\right)$

and relative index 0 if $i=0$, or relative index $-\sum_{j=1}^{i} k_{j}$ otherwise. Furthermore the critical values are related as follows:

$$
c_{0}^{-}<c_{1}^{-}<\cdots<c_{n}^{-}<0<c_{n}^{+}<\cdots<c_{1}^{+}<c_{0}^{+}
$$

Figure 10 illustrates one such construction for the link $(2,1,4)$.

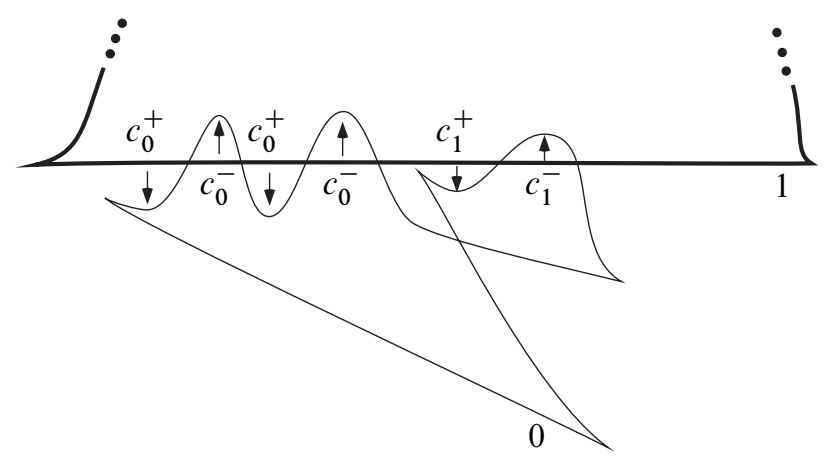

Figure 10: A portion of the Legendrian link $(2,1,4)$; the upper branch of $\Lambda_{1}$ is completed to have sufficiently positive slope on the left side so that its difference function $\Delta$ has $2(2+1)$ nondegenerate critical points, represented by pairs of points $\left(\left(x_{0}, 0, z_{1}\right),\left(x_{0}, 0, z_{0}\right)\right) \in \Lambda_{1} \times \Lambda_{0}$. In this figure, each pair is joined by a directed line segment, and the label ( $c_{0}^{ \pm}$or $\left.c_{1}^{ \pm}\right)$near each pair denotes the critical value. An initializing choice of branch labels is indicated.

Algebraic $8 \mathcal{G}$ Geometric Topology, Volume 6 (2006) 
Now for all $j, 1 \leq j \leq n$, choose noncritical values $a_{j}$ and $b_{j}$ such that

$$
c_{j-1}^{-}<a_{j}<c_{j}^{-}<0<c_{j}^{+}<b_{j}<c_{j-1}^{+} ;
$$

for $j=0$, we choose noncritical values $a_{0}, b_{0}$ satisfying

$$
a_{0}<c_{0}^{-}<0<c_{0}^{+}<b_{0} .
$$

For all $j$, we may apply a deformation argument as in the proof of Proposition 4.3 to construct a 1-parameter family of functions $\Delta_{t}$ such that $a_{j}, b_{j}$ are noncritical values of $\Delta_{t}$ for all $t \in[0,1], \Delta_{0}=\Delta$, and $\Delta_{1}$ has no critical points with values in $\left[a_{j}, b_{j}\right]$; this isotopy is not an isotopy of links but rather eliminates the $2 w_{n}+\cdots+2 w_{j}$ crossings. Thus by Lemma $4.4, H_{*}\left(\Delta_{0}^{b_{j}}, \Delta_{0}^{a_{j}}\right) \simeq H_{*}\left(\Delta_{1}^{b_{j}}, \Delta_{1}^{a_{j}}\right)=0$ for all $* \in \mathbb{Z}$. The theorem now follows from Lemma 5.3.

Theorem 5.4 (Remark 5.5) together with Theorem 4.11 (Remark 4.12) then prove

Corollary 5.6 Let $L=\left(\Lambda_{1}, \Lambda_{0}\right)$ be the rational Legendrian link

$$
\left(2 w_{n}, k_{n}, \ldots, 2 w_{1}, k_{1}, 2 w_{0}\right) \text {. }
$$

If the vector $\left(2 w_{n}, k_{n}, \ldots, 2 w_{1}, k_{1}, 2 w_{0}\right)$ is not palindromic, then the link $L$ is ordered.

Homology polynomials for "flypes" of links will also have a nice formulation. A vertical or horizontal flype is a move wherein a portion of a link is rotated $180^{\circ}$ about a vertical or horizontal axis (see Figure 11). For more background on flypes see, for example, Adams [1], Conway [5] or Traynor [19]. It is known that flypes produce topologically equivalent links, but we will use the polynomials to show that these flypes can produce nonequivalent Legendrian links.

Rational links give us many opportunities to apply flypes. As mentioned in the Introduction, we will only consider horizontal flypes since vertical flypes produce equivalent links. Given the rational link $L=\left(2 w_{n}, k_{n}, 2 w_{n-1}, \ldots, k_{1}, 2 w_{0}\right)$, we may apply one or more horizontal flypes using the horizontal crossings represented by the terms $2 w_{0}, 2 w_{1}, \ldots, 2 w_{n-1}$. Let $\left(2 w_{n}, k_{n}, 2 w_{n-1}^{p_{n-1}}, \ldots, k_{1}, 2 w_{0}^{p_{0}}\right)$ represent $L$ after it has undergone $p_{i}$ horizontal flypes using the set of $2 w_{i}$ horizontal crossings of $L$, where $0 \leq i \leq n-1$. See, for example, Figure 12, where the boxed portion of the link is the part rotated in the flype.

Theorem 5.7 Let $L=\left(2 w_{n}, k_{n}, 2 w_{n-1}^{p_{n-1}}, \ldots, k_{1}, 2 w_{0}^{p_{0}}\right)$. For $j=0,1, \ldots, n-1$, let $\sigma(j)=1+\sum_{i=0}^{j} p_{i} \bmod 2$. Then

$$
\Gamma^{-}(\lambda)[L]=\lambda^{m} \cdot\left[w_{0} \lambda^{0}+\sum_{i=1}^{n} w_{i} \lambda^{\left[(-1)^{\sigma(0)} k_{1}+\cdots+(-1)^{\sigma(i-1)} k_{i}\right]}\right],
$$




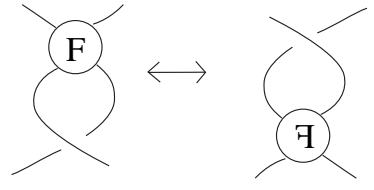

(a)

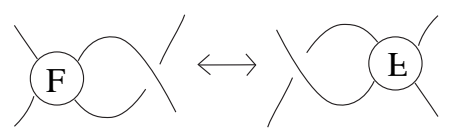

(b)

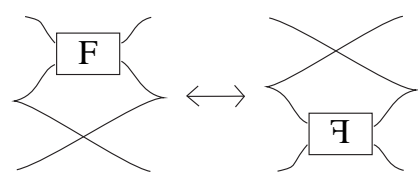

(c)

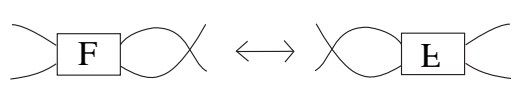

(d)

Figure 11: (a) a topological vertical flype, (b) a topological horizontal flype, (c) a Legendrian vertical flype, (d) a Legendrian horizontal flype

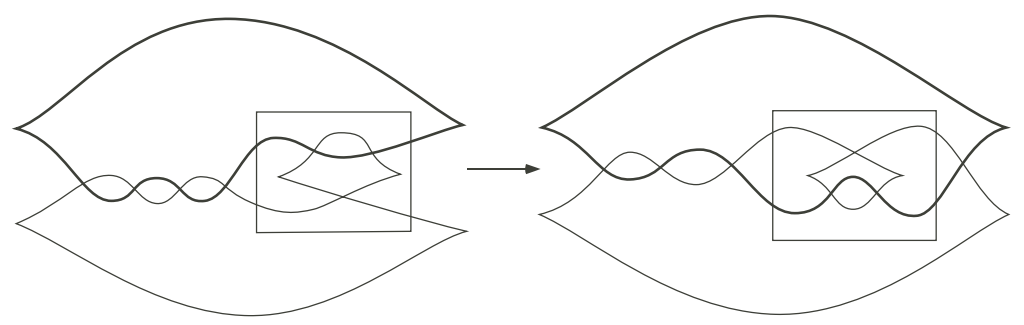

Figure 12: The rational link $(2,1,4)$ undergoes a horizontal flype and becomes $\left(2,1,4^{1}\right)$. These links have different negative homology polynomials.

where $m$ is chosen so that $\Gamma^{-}$has degree zero.

Remark 5.8 To construct the vector corresponding to the polynomial for $L=$ $\left(2 w_{n}, k_{n}, 2 w_{n-1}^{p_{n-1}}, \ldots, k_{1}, 2 w_{0}^{p_{0}}\right)$, one starts by writing a $w_{0}$ and then one goes backward (if $p_{0}$ is even) or forward (if $p_{0}$ is odd) $k_{1}$ places to write $w_{1}$. Then one repeats this procedure continuing in the same forward/backward direction as in the previous step if $p_{1}$ is even while changing direction if $p_{1}$ is odd, and adding the entries if one arrives at a position in the vector already visited. For example, if $L=(2,1,4)$ then the negative homology vector is $(1,2)$ (or, equivalently, $\Gamma^{-}(\lambda)[L]=2 \lambda^{0}+\lambda^{-1}$ ); after applying one horizontal flype, we have the link $L^{\prime}=\left(2,1,4^{1}\right)$ which has negative homology vector $(2,1)$ (equivalently $\Gamma^{-}(\lambda)\left[L^{\prime}\right]=\lambda^{0}+2 \lambda^{-1}$ ). So even though $L$ and $L^{\prime}$ are topologically equivalent and have the same classical Legendrian invariants, they are distinct Legendrian links; see Figure 12.

Proof This proof follows the proof of [19, Theorem 6.2]. We will give a sketch of the proof here. The key is to show the existence of a difference function $\Delta$ for $L$ such that $\Delta$ 
has $2\left(w_{0}+w_{1}+\cdots+w_{n}\right)$ nondegenerate critical points, and for $0 \leq i \leq n$ each of the $2 w_{i}$ critical points correspond to a pair of points $\left(\left(x_{0}, y_{0}, z_{1}\right),\left(x_{0}, y_{0}, z_{0}\right)\right) \in \Lambda_{1} \times \Lambda_{0}$ on branches $W_{1}^{i} \subset \Lambda_{1}, W_{0}^{i} \subset \Lambda_{0}$ where

(1) the distance function $d_{i}: \mathbb{R} \rightarrow \mathbb{R}$ given by $d_{i}(x)=\left|z_{1}(x)-z_{0}(x)\right|$, where $\left(x, y_{j}^{i}(x), z_{j}^{i}(x)\right) \in \Lambda_{j}$ is a point on branch $W_{j}^{i}$ for $j=0,1$, achieves a relative maximum at $x_{0}$ for each $i$ and

(2) $i_{B r}\left(W_{1}^{i}\right)-i_{B r}\left(W_{0}^{i}\right)= \begin{cases}0 & \text { if } i=0, \\ (-1)^{\sigma(0)} k_{1}+\cdots+(-1)^{\sigma(i-1)} k_{i} & \text { if } 1 \leq i \leq n .\end{cases}$

The existence of such a function is proven by induction, using the proof of Theorem 5.4 to prove the base case. Once we've proved the existence of a suitable difference function $\Delta$, the rest of the proof follows using the same reasoning as in the proof of Theorem 5.4.

Theorem 5.9 Let $L_{j, k}$ be a twist link as described in Figure 5. Then

$$
\Gamma^{-}(\lambda)\left[L_{j, k}\right]=\lambda^{0}+\lambda^{-|j-k|} .
$$

Remark 5.10 When $j \neq k$, the homology vector of $L_{j, k}$ is $(1,0, \ldots, 0,1)$ where there are $|j-k|-1$ zeros, while if $j=k$, the homology vector is (2).

Proof It is possible to isotope $L_{j, k}$ so that it has a difference function $\Delta: \mathbb{R} \times \mathbb{R}^{1+n_{1}} \times$ $\mathbb{R}^{1+n_{0}} \rightarrow \mathbb{R}$ with four nondegenerate critical points, as follows. The critical values of the critical points are $c_{1}^{+}, c_{1}^{-}$and $c_{0}^{+}, c_{0}^{-}$with the relative indices of the critical points (with an appropriate labeling of branch indices) 1,0 and $j-k+1, j-k$, respectively. Moreover the critical values satisfy the inequalities

$$
c_{0}^{-}<c_{1}^{-}<0<c_{1}^{+}<c_{0}^{+} .
$$

Figure 13 illustrates one such construction for the link $L_{2,3}$.

Choose noncritical values $a_{i}, b_{i}$ of $\Delta, i=0,1$, such that

$$
a_{0}<c_{0}^{-}<a_{1}<c_{1}^{-}<0<c_{1}^{+}<b_{1}<c_{0}^{+}<b_{0} .
$$

As in Theorem 5.4, we may deform $\Delta=\Delta_{0}$ (by pulling apart the bottom portions of the knots) so that $a_{1}, b_{1}$ are never critical values of $\Delta_{t}$, and $\Delta_{1}$ has no critical values in $\left[a_{1}, b_{1}\right]$. Again using Lemma 4.4, we conclude that $H_{*}\left(\Delta_{0}^{b_{1}}, \Delta_{0}^{a_{1}}\right) \simeq H_{*}\left(\Delta_{1}^{b_{1}}, \Delta_{1}^{a_{1}}\right)=$ 0 for all $* \in \mathbb{Z}$. Also notice that $H_{*}\left(\Delta^{b_{0}}, \Delta^{a_{0}}\right)=H_{*}(\Delta)=0$ by Proposition 4.3. So by Lemma 5.3,

$$
\Gamma^{-}(\lambda)\left[L_{j, k}\right]=\left(\lambda^{0}+\lambda^{(j-k)}\right) \cdot \lambda^{h},
$$

where $h$ is chosen so that $\Gamma^{-}(\lambda)\left[L_{j, k}\right]$ has degree zero. The result follows. 


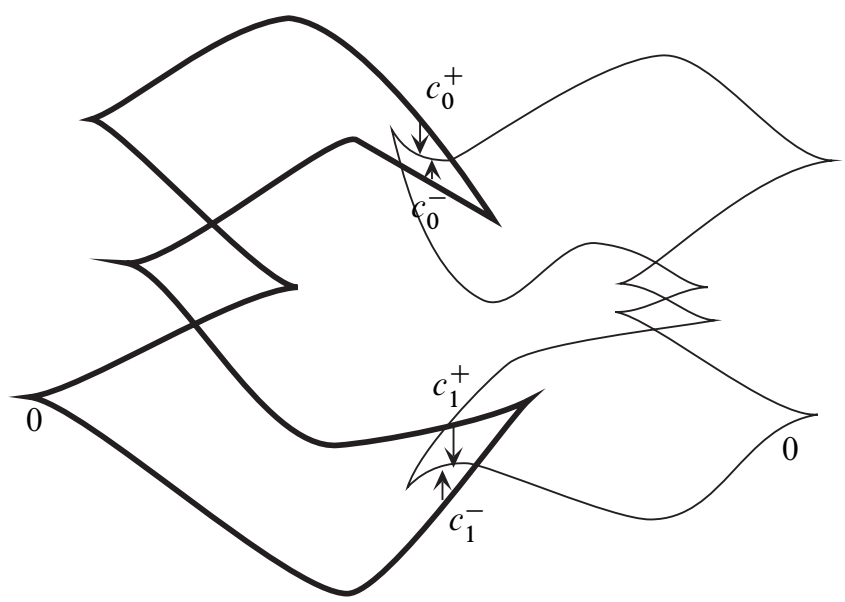

Figure 13: The Legendrian link $L_{2,3}$ can be positioned so that its difference function $\Delta$ has four nondegenerate critical points, represented by pairs of points $\left(\left(x_{0}, y_{0}, z_{1}\right),\left(x_{0}, y_{0}, z_{0}\right)\right) \in \Lambda_{1} \times \Lambda_{0}$. In this figure, each pair is joined by a line segment and the label $\left(c_{0}^{ \pm}\right.$or $\left.c_{1}^{ \pm}\right)$next to each pair indicates the critical value. An initializing choice of branch indices is indicated.

It is easy to see that $j+k$ determines the topological type of the link $L_{j, k}$. Theorem 5.9 then implies

Corollary 5.11 Let $m \geq 2$. If $m$ is even, the following links are topologically but not Legendrianly equivalent: $L_{1, m-1}, L_{2, m-2}, \ldots, L_{\frac{m}{2}}, \frac{m}{2}$. If $m$ is odd, the following links are not Legendrianly equivalent: $L_{1, m-1}, L_{2, m-2}, \ldots, L_{\frac{m-1}{2}}, \frac{m+1}{2}$.

\section{Comparison to other results}

In this section, we first compare the results of the homology polynomial calculations with various decomposition number invariants and then with a polynomial invariant that comes from a link's differential, graded algebra (DGA).

\subsection{Decomposition invariants}

There is a close relationship between generating families and the decomposition invariants of Chekanov [4], and Chekanov and Pushkar [14]. Briefly, an admissible decomposition of a front projection of a Legendrian link is a choice of the crossings in the front projection so that the front obtained by resolving all the crossings is a union 
of two cusped "eyes" that satisfy a set of conditions. For a precise definition, see [4]. In fact, as pointed out by Chekanov and Pushkar in [14, Section 12], the definition of an admissible decomposition is obtained by axiomatizing combinatorial structures arising on the front of a Legendrian submanifold defined by a generating family.

Chekanov showed that there are a number of decomposition invariants that can be associated to a Legendrian link $L$. For example, one can count the total number of admissible decompositions of any front of $L$. Also, one can count the total number of Maslov (also known as graded) admissible decompositions: one can associate a Maslov index to each branch of the front projection and then restrict to decompositions arising from resolving crossings of branches having the same Maslov index. One can also look at associated decomposition and Maslov decomposition polynomials: the decomposition polynomial is $D\left(L_{j, k}\right)=\sum_{\rho} z^{j(\rho)}$, where the sum is taken over all allowable decompositions $\rho$ of a front of $L_{j, k}$, and $j(\rho)$ is the number of left cusps in the decomposition minus the number of crossings resolved in the decomposition. The Maslov indices for the branches depend on a choice of initial value for a branch of each component, and different choices for the Maslov branch indices lead to different Maslov crossings for a 2 or more component link. Hence the Maslov decomposition number of a multi-component link is a set of decomposition numbers and the Maslov decomposition polynomial is a set of polynomials.

In our explorations, all these decomposition invariants are quite different than the invariants we have found through our use of generating families. As an illustration, we will just mention the decomposition invariants for the twist links.

Proposition 6.1 Let $L_{j, k}$ be the twist link; see Figure 5. Then

(1) for any $j, k$, the decomposition number of $L_{j, k}$ is 4 , and the decomposition polynomial of $L_{j, k}$ is $z^{-2}+2 z^{0}+z^{2}$;

(2) if $j=k$, the set of Maslov decomposition numbers of $L_{j, k}$ is $\{1,4\}$, and the set of Maslov decomposition polynomials equals $\left\{z^{2}, z^{-2}+2 z^{0}+z^{2}\right\}$; if $j \neq k$, the set of Maslov decomposition numbers of $L_{j, k}$ is $\{1,2\}$, and the set of Maslov decomposition polynomials equals $\left\{z^{2}, z^{0}+z^{2}\right\}$.

Proof First let us examine the non-Maslov decomposition numbers. Consider the front projection of $L_{j, k}$ as given by Figure 5. For any $j, k$, there are 4 allowable decompositions: one must resolve all the $j+k$ self-strand crossings, and then one has the choice to resolve none of the other crossings, the 2 uppermost crossings, the 2 lowermost crossings, or both the 2 uppermost and 2 lowermost crossings. For the 
particular front projection of $L_{j, k}$ as given by Figure 5, the number of left cusps is given by $j+k+2$, and thus we see that

$$
\begin{aligned}
D\left(L_{j, k}\right) & =z^{(j+k+2)-(j+k+0)}+2 z^{(j+k+2)-(j+k+2)}+z^{(j+k+2)-(j+k+4)} \\
& =z^{2}+2 z^{0}+z^{-2} .
\end{aligned}
$$

Now consider the Maslov versions. All the self-strand crossings are Maslov. When $j=k$, either none of the inter-strand crossings are Maslov, or all the crossings are Maslov. With the first type of labels, there is only one admissible decomposition. With the second type of labels, there are the same 4 as were seen above. Thus when $j=k$, the set of Maslov decompositions is $\{1,4\}$ and the Maslov decomposition polynomials are

$$
D^{\mu}\left(L_{j, k}\right)=\left\{z^{2}, z^{2}+2 z^{0}+z^{-2}\right\} .
$$

When $j \neq k$, there are essentially three ways to label the branches: either none of the inter-strand crossings are Maslov, or only the top two inter-strand crossings are Maslov, or only the bottom two inter-strand crossings are Maslov. With the first type of labels, we have one admissible decomposition. With either the second or third type of labels, we have two admissible decompositions. Hence the set of Maslov decomposition numbers is $\{1,2\}$. The set of associated Maslov decomposition polynomials is

$$
D^{\mu}\left(L_{j, k}\right)=\left\{z^{2}, z^{2}+z^{0}\right\} .
$$

See Figure 14.

In particular, we see that these decomposition invariants cannot distinguish, for example, the topologically equivalent Legendrian links $L_{1,4}$ and $L_{2,3}$ which can be distinguished by the generating family polynomials. It would be interesting to see if the decomposition invariants can be further refined so that they do capture the same information as the generating family polynomials.

\subsection{DGA invariants}

First we will briefly outline the process by which a differential, graded algebra (DGA) is associated to a link, and a polynomial is in turn associated to the algebra. This set-up is analogous to the work of $\mathrm{Ng}$ [12].

The first step is to associate an algebra to the front projection of a link. The algebra $\mathcal{A}$ is given to be the free, unital, associative algebra with coefficients in $\mathbb{Z} / 2 \mathbb{Z}$ generated by the crossings and right cusps of the front projection of the link. Each generator is assigned a degree, which extends to all of $\mathcal{A}$ by multiplicativity. The degree of each cusp is 1 , while the degree of each crossing is calculated using a simple procedure 


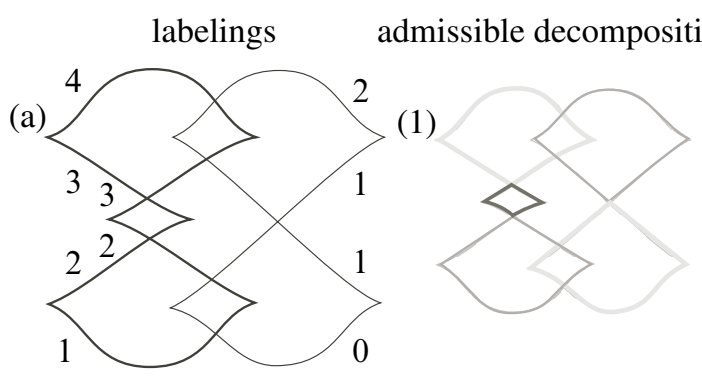

(b)

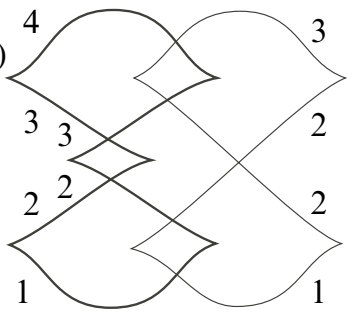

(1)

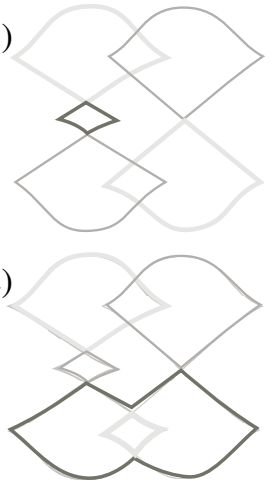

Figure 14: In (a), the branches of $L_{2,1}$ are labeled so that no inter-strand crossings are Maslov. The unique Maslov admissible decomposition is shown. In (b), the branches are labeled so that only the bottom set of inter-strand crossings are Maslov. The two Maslov admissible decompositions are shown. Note that a third labeling is possible, in particular one in which only the top set of inter-strand crossings are Maslov. The two admissible decompositions in that case are symmetric to those in the second labeling.

described in [12, Section 2.2]. The degree depends upon a choice of marked points on each component of the link: a change in the choice of marked points may shift some of the degrees by an integer. In $\mathrm{Ng}$ and Traynor [13], there was a canonical choice for the marked points, but not for the links in $\mathbb{R}^{3}$ that we are considering.

The second step is to calculate a map $\partial: \mathcal{A} \rightarrow \mathcal{A}$, called a differential, having the properties that $\partial^{2}=0$ and $\partial$ lowers degree by one. $\partial$ is defined over $\mathbb{Z} / 2 \mathbb{Z}$ on the generators of $\mathcal{A}$ by counting certain immersed disks in the front projection. For details, see [12, Section 2.2].

In order to use the DGA to distinguish links, we use additional structure properties described in $[13,2.4]$, which is essentially Mishachev's relative homotopy splitting from [11]. We break up $\mathcal{A}$ into components $\mathcal{A}^{1,1}, \mathcal{A}^{0,0}, \mathcal{A}^{1,0}$, and $\mathcal{A}^{0,1}$, where the idea is to separate the generators based on how strands from the link components $\Lambda_{1}$ 
and $\Lambda_{0}$ contribute to each generator. For example, $\mathcal{A}^{1,0}$ is the module over $\mathbb{Z} / 2 \mathbb{Z}$ generated by words of the form $a_{i_{1}} \cdots a_{i_{m}}$ where the overstrand of $a_{i_{1}}$ is in $\Lambda_{1}$ and the understrand of $a_{i_{m}}$ is in $\Lambda_{0}$, and the understrand of $a_{i_{p}}$ is in the same link component as the overstrand of $a_{i_{p+1}} \cdot \mathcal{A}^{1,1}\left(\mathcal{A}^{0,0}\right)$ is generated by analogous words together with an indeterminate $e_{1}\left(e_{0}\right)$. The map $\partial$ is altered to be defined on $\mathcal{A}^{i, j}$, and we call the new differential $\partial^{\prime}$. On $\mathcal{A}^{1,0}$ and $\mathcal{A}^{0,1}, \partial^{\prime}$ agrees with $\partial$, while on $\mathcal{A}^{1,1}\left(\mathcal{A}^{0,0}\right)$ there is the slight modification that any 1 term is replaced by $e_{1}\left(e_{0}\right)$. Thus we have a link DGA given by $\left(\mathcal{A}^{*, *}, \partial^{\prime}\right)$, and explicitly defined in [13, Definition 2.13].

Once we have a link DGA for a link $L$, we may use it to calculate a polynomial invariant of $L$, up to equivalence. A full description of this process is given in [12, Section 2.2] or [13, 2.5]. It involves defining an augmentation $\epsilon$, which is a map from $\mathcal{A}$ to $\mathbb{Z} / 2 \mathbb{Z}$. Using this augmentation, we calculate $\partial_{\epsilon}^{\prime}: V_{k}^{i, j} \rightarrow V_{k-1}^{i, j}$, where $V_{k}^{i, j}$ is the graded vector space over $\mathbb{Z} / 2 \mathbb{Z}$ generated by the generators of $\mathcal{A}^{i, j}$ of degree $k$. In particular, $V_{k}^{1,0}$ is generated by degree $k$ crossings with an overstrand in $\Lambda_{1}$ and an understrand in $\Lambda_{0}$. Define

$$
\beta_{k}^{1,0}(\epsilon)=\operatorname{dim} \frac{\operatorname{ker} \partial_{\epsilon}^{\prime}: V_{k}^{1,0} \rightarrow V_{k-1}^{1,0}}{\operatorname{im} \partial_{\epsilon}^{\prime}: V_{k+1}^{1,0} \rightarrow V_{k}^{1,0}}
$$

and define

$$
\chi_{\epsilon}^{1,0}(\lambda)[L]=\sum_{k} \beta_{k}^{1,0}(\epsilon) \cdot \lambda^{k} .
$$

Then $\chi_{\epsilon}^{1,0}$ is one of the split Poincaré-Chekanov polynomials of $L$ with respect to $\epsilon$. Polynomials $\chi_{\epsilon}^{1,1}, \chi_{\epsilon}^{0,0}$, and $\chi_{\epsilon}^{0,1}$ can be defined similarly. The polynomials $\chi_{\epsilon}^{1,0}$ and $\chi_{\epsilon}^{0,1}$ depend on the grading of the link; in particular, changing the choice of marked points on the components has the potential to shift the degree of the polynomials up or down by some integer. We will focus on normalized versions of the $\chi_{\epsilon}^{1,0}$ polynomials. Therefore we will define the normalized negative Poincaré-Chekanov polynomial as

$$
\chi_{\epsilon}^{-}=\chi_{\epsilon}^{1,0} \cdot \lambda^{m},
$$

where $m$ is chosen so that $\chi_{\epsilon}^{-}$has degree 0 . The set $\left\{\chi_{\epsilon}^{-}\right\}$arising from different choices of $\epsilon$ is an invariant of a Legendrian link $\left(\Lambda_{1}, \Lambda_{0}\right)$. In the cases studied in this paper, there will always be a unique polynomial and so the set notation will be dropped.

We first compute the normalized negative Poincaré-Chekanov polynomial of a Legendrian rational link; compare Theorem 5.4.

Theorem 6.2 Let $L=\left(2 w_{n}, k_{n}, \ldots, 2 w_{1}, k_{1}, 2 w_{0}\right)$ (see Figure 9). Then

$$
\chi_{\epsilon}^{-}(\lambda)[L]=w_{0} \lambda^{0}+w_{1} \lambda^{-k_{1}}+w_{2} \lambda^{-\left(k_{1}+k_{2}\right)}+\cdots+w_{n} \lambda^{-\left(k_{1}+k_{2}+\cdots+k_{n}\right)} .
$$


Proof We use $c_{i}$ 's and $v_{i}$ 's to denote generators arising from right cusps and vertical crossings, respectively. Let $h_{1}, h_{2}, \ldots, h_{2} \sum w_{i}$ be the $2 \sum_{i=0}^{n} w_{i}$ generators arising from the horizontal crossings, numbered right to left. For example, Figure 15 shows the generators for $L=(2,1,4,2,2)$.

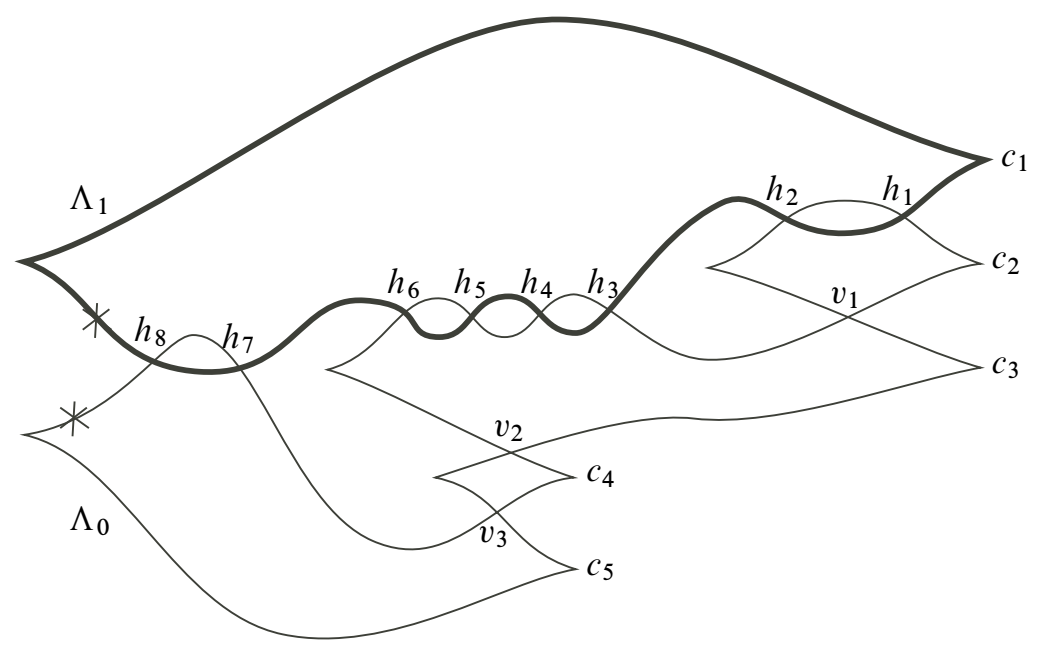

Figure 15: The generators of $\mathcal{A}$ for $L=(2,1,4,2,2)$ are labeled using $c_{i}$ 's, $v_{i}$ 's, and $h_{i}$ 's, and the $*$ 's on each component of $L$ indicate the choice of marked points.

Since we are calculating $\chi_{\epsilon}^{-}$, we focus on generators in $V^{1,0}$, namely $\left\{h_{m} \mid m\right.$ is even $\}$. We can choose marked points so that the degrees of the $h_{m}$ are as follows:

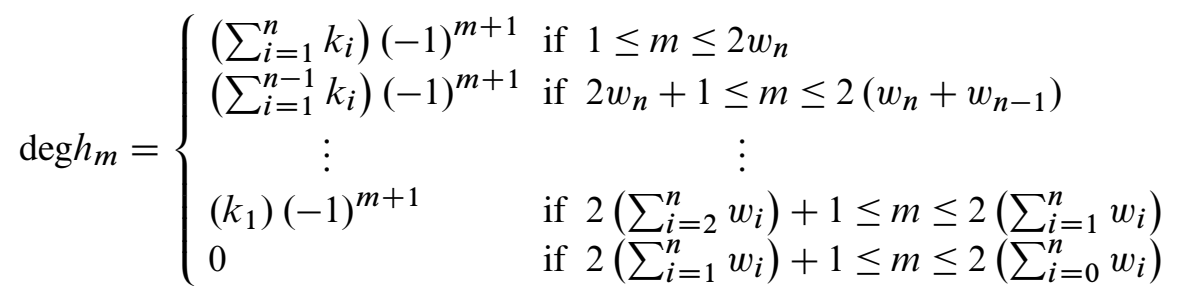

For example, in the link $L=(2,1,4,2,2)$ with marked points as indicated in Figure 15 , we have the following:

$$
\operatorname{deg} h_{m}= \begin{cases}3 & \text { if } m=1 \\ -3 & \text { if } m=2 \\ 2 & \text { if } m=3,5 \\ -2 & \text { if } m=4,6 \\ 0 & \text { if } m=7,8\end{cases}
$$


This link has a unique augmentation $\epsilon$, for which $\epsilon\left(h_{m}\right)=0$ for all $m$. Each $h_{m}$ is in the kernel of the $\partial_{\epsilon}^{\prime}$ map of the appropriate degree, but no $h_{m}$ s are in the image of any $\partial_{\epsilon}^{\prime}$ map. Thus we have

$$
\chi_{\epsilon}^{1,0}(\lambda)[L]=\sum_{j=-\infty}^{\infty} N\left(h_{m}, j\right) \cdot \lambda^{j},
$$

where $N\left(h_{m}, j\right)$ is the number of generators $h_{m} \in \mathcal{A}^{1,0}$ of degree $j$. Hence, checking the degrees of the $h_{m}$ given above, we have

$$
\chi_{\epsilon}^{1,0}(\lambda)[L]=w_{0} \lambda^{0}+w_{1} \lambda^{-\left(k_{1}\right)}+w_{2} \lambda^{-\left(k_{1}+k_{2}\right)}+\cdots+w_{n} \lambda^{-\left(k_{1}+k_{2}+\cdots+k_{n}\right)} .
$$

Since this polynomial is of degree 0 , it is in fact equal to $\chi_{\epsilon}^{-}$.

Lastly, we compute the normalized negative Poincaré-Chekanov polynomial of a Legendrian twist link; compare Theorem 5.9.

Theorem 6.3 Let $L_{j, k}$ be the twist link described in Figure 5. Then

$$
\chi_{\epsilon}^{-}(\lambda)\left[L_{j, k}\right]=\lambda^{0}+\lambda^{-|j-k|} .
$$

Proof Use $c_{i}$ 's and $s_{i}$ 's to denote generators arising from the right cusps and selfstrand crossings of $L_{j, k}$, respectively. Use $x_{1}, x_{2}, x_{3}$, and $x_{4}$ to denote generators arising from the four interstrand crossings, numbered from top to bottom. See Figure 16 for an example of how to label generators.

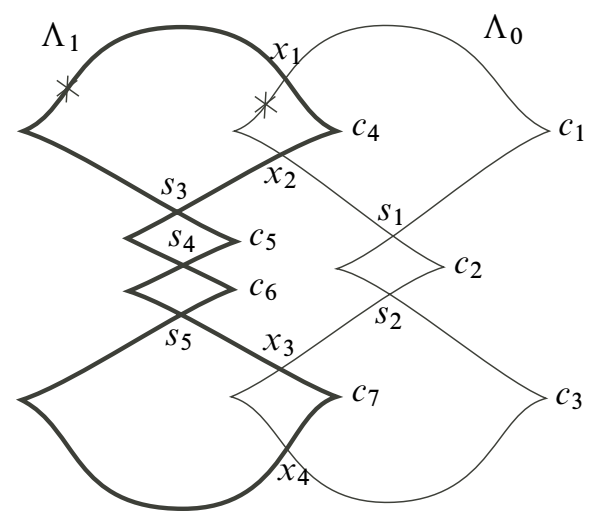

Figure 16: Labeling of generators of $\mathcal{A}$ for the link $L_{3,2}$, where the $*$ 's on each component of $L_{3,2}$ indicate the choice of marked points

Note that $\mathcal{A}^{1,0}$ is generated by $\left\{x_{1}, x_{3}\right\}$. We can choose marked points so that $\operatorname{deg}\left(x_{1}\right)=0$ and $\operatorname{deg}\left(x_{3}\right)=k-j$. We have a unique augmentation $\epsilon$. Calculating $\partial_{\epsilon}^{\prime}$, 
we find that both $x_{1}$ and $x_{3}$ are in the kernel of the $\partial_{\epsilon}^{\prime}$ map of the appropriate degree, and neither is in the image of a $\partial_{\epsilon}^{\prime}$ map. Thus we have

$$
\chi_{\epsilon}^{1,0}(\lambda)\left[L_{j, k}\right]=\lambda^{0}+\lambda^{k-j} .
$$

The formula for the normalized Poincaré-Chekanov polynomial follows.

Summarizing the results from Theorems 5.4, 6.2, 5.9, and 6.3, we find:

Theorem 6.4 Let $L$ be a Legendrian link that is either a rational link or a twist link. Then the negative homology polynomial of $L$ is the same as its normalized negative Poincaré-Chekanov polynomial.

\section{References}

[1] C C Adams, The knot book, W H Freeman and Company, New York (1994) MR1266837

[2] V I Arnol'd, S M Guseĭn-Zade, A N Varchenko, Singularities of differentiable maps. Vol. I, Monographs in Mathematics 82, Birkhäuser, Boston (1985) MR777682

[3] Y Chekanov, Differential algebra of Legendrian links, Invent. Math. 150 (2002) 441483 MR1946550

[4] Y V Chekanov, Invariants of Legendrian knots, from: "Proceedings of the International Congress of Mathematicians, Vol. II (Beijing, 2002)”, Higher Ed. Press, Beijing (2002) 385-394 MR1957049

[5] J H Conway, An enumeration of knots and links, and some of their algebraic properties, from: "Computational Problems in Abstract Algebra (Proc. Conf., Oxford, 1967)", Pergamon, Oxford (1970) 329-358 MR0258014

[6] Y Eliashberg, A Givental, H Hofer, Introduction to symplectic field theory, Geom. Funct. Anal. (2000) 560-673 MR1826267

[7] Y Eliashberg, M Gromov, Lagrangian intersection theory: finite-dimensional approach, from: "Geometry of differential equations", Amer. Math. Soc. Transl. Ser. 2 186, Amer. Math. Soc., Providence, RI (1998) 27-118 MR1732407

[8] C Ernst, D W Sumners, A calculus for rational tangles: applications to DNA recombination, Math. Proc. Cambridge Philos. Soc. 108 (1990) 489-515 MR1068451

[9] J B Etnyre, Legendrian and transversal knots, from: "Handbook of knot theory", Elsevier B. V., Amsterdam (2005) 105-185 MR2179261

[10] J B Etnyre, L L Ng, J M Sabloff, Invariants of Legendrian knots and coherent orientations, J. Symplectic Geom. 1 (2002) 321-367 MR1959585 
[11] K Mishachev, The $N$-copy of a topologically trivial Legendrian knot, J. Symplectic Geom. 1 (2003) 659-682 MR2039159

[12] L L Ng, Computable Legendrian invariants, Topology 42 (2003) 55-82 MR1928645

[13] L Ng, L Traynor, Legendrian solid-torus links, J. Symplectic Geom. 2 (2004) 411-443 MR2131643

[14] P E Pushkar', Y V Chekanov, Combinatorics of fronts of Legendrian links, and Arnol'd's 4-conjectures, Uspekhi Mat. Nauk 60 (2005) 99-154 MR2145660

[15] D Théret, A complete proof of Viterbo's uniqueness theorem on generating functions, Topology Appl. 96 (1999) 249-266 MR1709692

[16] D Théret, A Lagrangian camel, Comment. Math. Helv. 74 (1999) 591-614 MR1730659

[17] L Traynor, Symplectic homology via generating functions, Geom. Funct. Anal. 4 (1994) 718-748 MR1302337

[18] L Traynor, Legendrian circular helix links, Math. Proc. Cambridge Philos. Soc. 122 (1997) 301-314 MR1458235

[19] L Traynor, Generating function polynomials for Legendrian links, Geom. Topol. 5 (2001) 719-760 MR1871403

[20] C Viterbo, Symplectic topology as the geometry of generating functions, Math. Ann. 292 (1992) 685-710 MR1157321

Department of Mathematics, Bryn Mawr College

Bryn Mawr PA 19010, USA

Department of Mathematics, Bryn Mawr College

Bryn Mawr PA 19010, USA

jill.e.jordan@hotmail.com, ltraynor@brynmawr.edu

Received: 28 March 2006

Algebraic $6 \mathcal{G}$ Geometric Topology, Volume 6 (2006) 